\title{
Article \\ Extended fMRI-Guided Anodal and Cathodal Transcranial Direct Current Stimulation Targeting Perilesional Areas in Post-Stroke Aphasia: A Pilot Randomized Clinical Trial
}

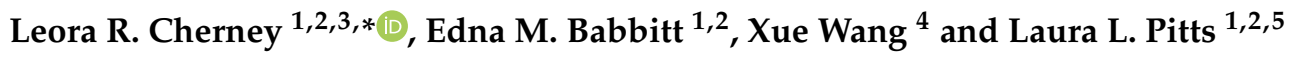 \\ 1 Think + Speak Lab, Shirley Ryan AbilityLab, Chicago, IL 60611, USA; ebabbitt@sralab.org (E.M.B.); \\ laura.pitts@uni.edu (L.L.P.) \\ 2 Department of Physical Medicine and Rehabilitation, Feinberg School of Medicine, Northwestern University, \\ Chicago, IL 60611, USA \\ 3 Department of Communication Sciences and Disorders, Northwestern University, Evanston, IL 60208, USA \\ 4 Department of Radiology, Feinberg School of Medicine, Northwestern University, Chicago, IL 60611, USA; \\ xuewang2@gmail.com \\ 5 Department of Communication Sciences and Disorders, University of Northern Iowa, \\ Cedar Falls, IA 50614, USA \\ * Correspondence: lcherney@sralab.org
}

check for updates

Citation: Cherney, L.R.; Babbitt, E.M.; Wang, X.; Pitts, L.L. Extended fMRI-Guided Anodal and Cathodal Transcranial Direct Current Stimulation Targeting Perilesional Areas in Post-Stroke Aphasia: A Pilot Randomized Clinical Trial. Brain Sci. 2021, 11, 306. https://doi.org/ 10.3390/brainsci11030306

Academic Editor: Paola Marangolo

Received: 23 December 2020

Accepted: 25 February 2021

Published: 28 February 2021

Publisher's Note: MDPI stays neutral with regard to jurisdictional claims in published maps and institutional affiliations.

Copyright: (c) 2021 by the authors. Licensee MDPI, Basel, Switzerland. This article is an open access article distributed under the terms and conditions of the Creative Commons Attribution (CC BY) license (https:// creativecommons.org/licenses/by/ $4.0 /)$.
Abstract: Transcranial direct current stimulation (tDCS) may enhance speech and language treatment (SLT) for stroke survivors with aphasia; however, to date, there is no standard protocol for the application of $\mathrm{tDCS}$ in post-stroke aphasia. We explored the safety and efficacy of fMRI-guided tDCS on functional language and cortical activity when delivered to the lesioned left hemisphere concurrently with SLT across an extended, six-week treatment period. Twelve persons with chronic, nonfluent aphasia following a single left-hemisphere stroke participated in the three-arm (anodal vs. cathodal vs. sham) single-blind, parallel, pilot trial. No serious adverse events occurred during 30 treatment sessions or in the following six weeks. All groups demonstrated functional language gains following intensive treatment; however, active tDCS resulted in greater gains in standardized, probe, and caregiver-reported measures of functional language than sham. Evidence declaring one polarity as superior for inducing language recovery was mixed. However, cathodal stimulation to the lesioned left hemisphere, expected to have a down-regulating effect, resulted in increased areas of cortical activation across both hemispheres, and specifically perilesionally. Generalization of these preliminary findings is limited; however, results are nevertheless compelling that $\mathrm{tDCS}$ combined with SLT can be safely applied across extended durations, with the potential to enhance functional language and cortical activation for persons with aphasia.

Keywords: transcranial direct current stimulation (tDCS); aphasia; speech language therapy; noninvasive brain stimulation (NIBS); nonfluent; interhemispheric inhibition (IHI)

\section{Introduction}

Approximately one-third of the 104 million stroke survivors worldwide live with resultant aphasia, or difficulty comprehending and/or expressing language [1,2]. Persons with aphasia often experience significant secondary disability including depression, isolation, decreased quality of life, and difficulty resuming everyday life activities including returning to work [3-7]. Despite advances in gold-standard behavioral speech-language therapy (SLT), the recovery of functional communication is often slow with minimal to moderate gains $[8,9]$. Transcranial direct current stimulation (tDCS) may bolster aphasia recovery when combined with SLT; however, optimal parameters for tDCS protocols to rehabilitate aphasia remain unclear due to differences across studies in research designs, tDCS protocols, and participant characteristics [10-13]. The current evidence is insufficient to (1) determine which polarity (anodal, cathodal, or dual) and stimulation site is most 
efficacious, (2) establish safety across extended use, and (3) fully characterize the impact of adjuvant tDCS on functional language outcomes $[10,11]$.

In tDCS, a mobile battery-operated direct current stimulator delivers a constant, weak direct current between two surface electrodes on the scalp. One electrode is placed on the site overlying the cortical target and a reference electrode is placed over an area considered to be remote from the cortical target such as the contralateral supraorbital area, contralateral mastoid, or contralateral shoulder. The nature of the effect depends on the polarity of the current, i.e., the direction of the flow of the current. In general, it is assumed that anodal tDCS results in increased cortical excitability whereas cathodal tDCS decreases cortical excitability [14-16].

Across the aphasia literature, anodal stimulation (A-tDCS) to the lesioned hemisphere has been most often investigated with the intent of promoting perilesional activation whereas cathodal stimulation (C-tDCS) has been applied to the intact, homologous cortex to downregulate activity largely based on the theory of interhemispheric competition $[10,17,18]$. However, there is growing concern that suppressing interhemispheric inhibition (IHI) in the contralesional hemisphere may be an oversimplified or erroneous model of functional reorganization after a stroke [18].

Studies investigating cathodal stimulation to the lesioned left hemisphere are rare. Interestingly, the first investigation of tDCS in aphasia rehabilitation reported improved naming accuracy following a single session of cathodal stimulation over the left frontotemporal cortex (without concurrent SLT) across eight stroke survivors with non-fluent aphasia [19]. More recently, cathodal (C-tDCS) stimulation to the lesioned hemisphere proved to be the most consistent montage to immediately increase naming accuracy when compared to a sham condition and additional permutations of either A-tDCS or C-tDCS to each hemisphere [20]. However, to our knowledge, there have been no other reports of cathodal stimulation to the left lesioned hemisphere in persons with aphasia.

In addition to decisions regarding selection of the cortical hemisphere and the polarity of stimulation, there is insufficient information about the specific stimulation target. In many studies, the stimulation site is selected apriori and identified using the 10-20 EEG measurement system in the absence of neuroimaging data. However, selecting a common site for all participants without knowledge of a participant's specific lesion location and size may result in electrodes being placed on tissue that is not viable, thereby altering the flow of the current and subsequent effects of the tDCS [21,22]. Other studies have completed imaging prior to $\mathrm{tDCS}$ to ensure that the electrodes are placed over surviving viable tissue [23], but have not individualized the electrode location based on the functional integrity of the tissue. Selecting a stimulation site based on the individual's specific anatomic and functional neuroimaging data may lead to more consistent results within and across studies [12]. Thus, the present heterogeneity of applied montages across investigations precludes the development of guidelines for tDCS applications to treat post-stroke aphasia.

Furthermore, a recent 2019 Cochrane Review of tDCS applications in post-stroke aphasia rehabilitation concluded that although no serious, harmful effects of tDCS have been published, further trials are needed to determine the safety of tDCS, especially when applied over extended durations such as those similar to clinical intervals of SLT [10]. The same Cochrane Review and others also noted that there is no current consensus that tDCS may enhance aphasia recovery beyond improved accuracy in naming nouns [10,13]. Indeed, the majority of studies have paired the tDCS with some form of naming therapy that is not always evidence-based [13,24]. Thus, a critical question remains: Does adjuvant tDCS have any therapeutic use in aphasia rehabilitation aside from pairing with single word naming treatments, especially without consensus for any clinically meaningful gains in functional language?

Here, we explored the effects of an extended, six-week application of perilesional A-tDCS, C-tDCS, or sham (S-tDCS) stimulation with concurrent SLT on language skills and cortical activation maps in nonfluent aphasia. The specific aims were to assess whether prolonged administration of tDCS is safe, and if a single polarity targeting the lesioned 
left hemisphere improves and/or maintains gains in language to a greater extent than sham stimulation and the opposing polarity. Results may substantially shift paradigms that most often perpetuate anodal and largely ignore cathodal stimulation to the lesioned hemisphere. Data supporting extended tolerability and potential for functional language gains will reinforce the promise of feasibility and efficacy of adjuvant tDCS applications in routine aphasia rehabilitation.

\section{Materials and Methods}

This Phase 1 single-blind placebo-controlled randomized clinical pilot study was approved on 5/8/2009 by the Institutional Review Board at Northwestern University (STU00011132) and subsequently allocated participants across three parallel arms with an allocation ratio of 1:1:1 (A-tDCS: C-tDCS: S-tDCS). The study was conducted in accordance with the Declaration of Helsinki and registered as a clinical trial (NCT01486654). All subjects provided informed consent prior to participation. No interim analyses or stopping guidelines were enacted or required by protocol.

\subsection{Participants}

Participants were enrolled from May 2010 to December 2012 at an urban rehabilitation center. Inclusion criteria were as follows: men or women with a diagnosis of non-fluent aphasia subsequent to a single-event, unilateral left hemisphere stroke that occurred more than six months prior to participation and was confirmed by CT scan or MRI; an Aphasia Quotient on the Western Aphasia Battery-Revised (WAB-R AQ) of 25-75 [25]; premorbidly literate in English; right-hand dominant as determined by the Edinburgh Handedness Inventory [26]; at least an eighth grade education; current visual acuity no worse than 20/100 corrected in the better eye; auditory acuity no worse than $30 \mathrm{~dB}$ HL on a pure tone hearing screening (conducted at 500, 1000, 2000, and $4000 \mathrm{~Hz}$ ), aided in the better ear; seizure-free for six months or longer prior to study enrollment. Participants were excluded if they had a neurological condition other than cerebral vascular disease that could potentially affect cognition or speech (e.g., Parkinson's disease, Alzheimer's dementia, traumatic brain injury); significant psychiatric history (e.g., severe depression or psychotic disorder requiring hospitalization); or contraindications for magnetic resonance imaging (MRI). A sample size of 12 participants (four in each arm) was considered to be feasible given the exploratory nature of the study and its financial and time constraints.

\section{2. fMRI-Guided Localization of Perilesional Stimulation Site}

Structural and functional scans were obtained on a Siemens 3T TIM Trio scanner (Siemens Medical Solutions, Erlangen, Germany) with a 32-channel head coil at two time points: before and after six weeks of SLT paired with tDCS. Pre-treatment scans were used to determine eligibility, establish baseline patterns of brain activation during language tasks, and guide the identification of individualized stimulation sites for tDCS. Post-treatment scans assessed physiological changes associated with treatment. T1-weighted anatomical images were acquired with TR/TE of $2300 / 2.97 \mathrm{~ms}$, flip angle of $9^{\circ}$, matrix resolution of $176 \times 256 \times 256$, and voxel size of $1 \times 1 \times 1 \mathrm{~mm}$. Functional tasks were acquired with highresolution gradient echo-planar imaging (EPI) data, TR/TE of 2200/20 ms, flip angle of $80^{\circ}$, in-plane resolution of $128 \times 116$, voxel size of $1.72 \times 1.72 \times 3 \mathrm{~mm}$ across a total of 37 slices for whole brain coverage. Tasks included: 1 . semantic categorization, 2 . oral reading of a word within a sentence, and 3. imitation of consonant-vowel syllables. All three tasks were pre-trained within three days prior to the fMRI evaluation, then retrained just prior to entering the MRI.

Task 1 involved a semantic decision. Participants were shown two words aligned vertically on the screen. The top word was a category name (e.g., flower) and directly underneath was an object label (e.g., either rose or chair). Using their unimpaired, left hand, participants pressed one of two buttons on a fiberoptic device shaped like a computer mouse to indicate either "yes" or "no" regarding whether or not the object label belonged 
to the above category. Each pairing was displayed for up to five seconds or until a response was made. Task 1 included eight blocks (i.e., $48 \mathrm{~s}$ in duration with eight pairs of words presented in random order) interleaved with eight rest blocks (i.e., $48 \mathrm{~s}$ in duration during which the participant was asked to focus on a cross displayed in the middle of the screen). The total time for Task 1 was $10 \mathrm{~min}, 25 \mathrm{~s}$.

Task 2 required participants to read aloud single words (i.e., oral reading). Participants were shown three- to five-word sentences on the screen for a total of six seconds each. For half of the presented sentences, one of the content words changed from black font to red font after two seconds and stayed red for the remaining four seconds. For the other half of the presented sentences, all words remained in black font while displayed for six seconds. Participants were asked to read a single word aloud only if it turned red and to remain silent if no color changes occurred. During Task 2, there were six overt speech blocks interleaved with six covert blocks. Each block contained four short sentences. The total time for Task 2 was $8 \mathrm{~min}, 40 \mathrm{~s}$.

During Task 3, participants watched short, $1.5 \mathrm{~s}$ video clips of a woman producing consonant-vowel syllables (e.g., pa, fa, ta, and $\theta \mathrm{a}$ ) and were required to imitate the syllables. Task 3 was an event-related design with jittered intertrial intervals. The total time for Task 3 was $10 \mathrm{~min}, 39 \mathrm{~s}$.

These fMRI tasks were selected because they required skills necessary for the behavioral treatment provided concurrently with the tDCS (i.e., matching words, reading aloud words within sentences, and observation and imitation of oral motor movements). It was expected that these fMRI tasks would subsequently recruit cortical regions required for the behavioral treatment such as premotor cortex (including the pars opercularis of the inferior frontal gyrus), superior temporal gyrus and occipito-temporal cortex-regions thought to be particularly important in speech production, language comprehension and reading, respectively $[27,28]$. Furthermore, these tasks had been used in previous studies with aphasia, including one study where common areas of cortical activation in the left hemisphere were used successfully to identify the site for epidural cortical stimulation [27-29].

The stimulation site, or fMRI-navigated electrode placement, was defined a priori and determined as overlapping left hemisphere areas of cortical activation (i.e., BOLD signal) across at least two of the three tasks for each individual. fMRI images were overlaid onto 2D MR cross-sectional images and a 3D reconstructed head image, and co-registered with external cranial landmarks to generate a scalp projection using the eXimia neuronavigation system (Nexstim Ltd., Helsinki, Finland). The scalp projection of the stimulation site was marked on the participant with indelible ink, which was maintained throughout the treatment interval and calibrated between the neuronavigation system and manual tape measurements using external landmarks (e.g., tragus of the outer ear, nasion). For all participants, periodic validation of electrode placement was conducted within the first two weeks of treatment using the neuronavigation system. Figure 1 illustrates the process of localizing the fMRI-guided stimulation site using the neuronavigation system. Figure 2 illustrates examples of perilesional electrode location and orientation for three participants (i.e., the first participant randomized to each arm). The orientation of the active electrode was selected so that the greatest area of activation was covered. Figure 3 illustrates the fMRI-guided stimulation sites for all study participants plotted in standard Montreal Neurological Institute (MNI) space on a normal model of the left hemisphere. Table 1 reports each individual's stimulation site by Harvard-Oxford Brain Atlas classifications. For all participants, the reference electrode was placed horizontally over the right contralateral supraorbital area. 


\section{A. 2D fMRI}

Task 1

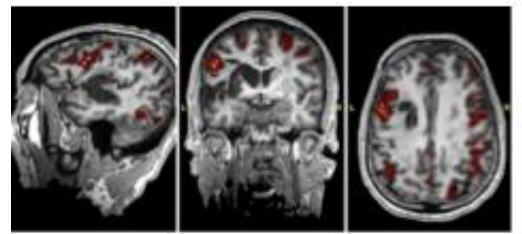

B. 3D fMRI

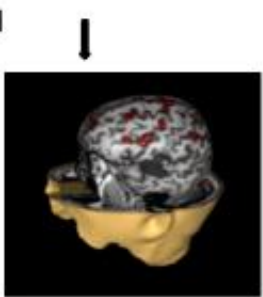

C. Scalp Targets
Task 2

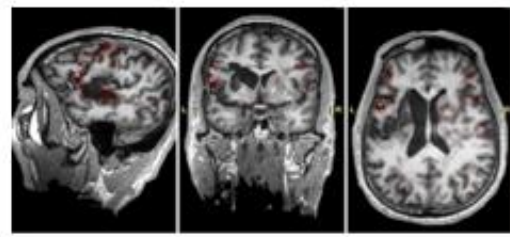

I
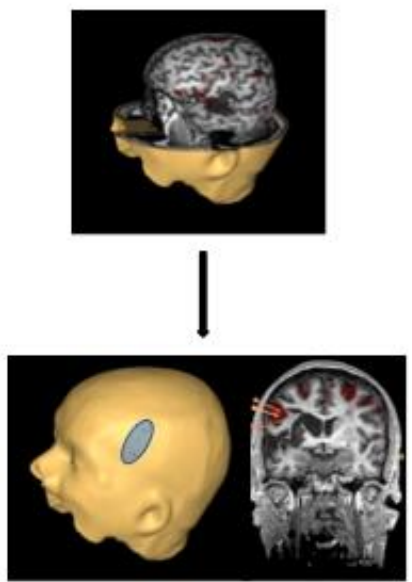

Task 3

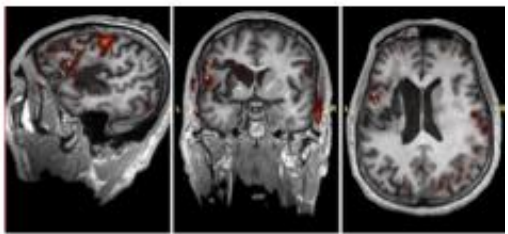

I
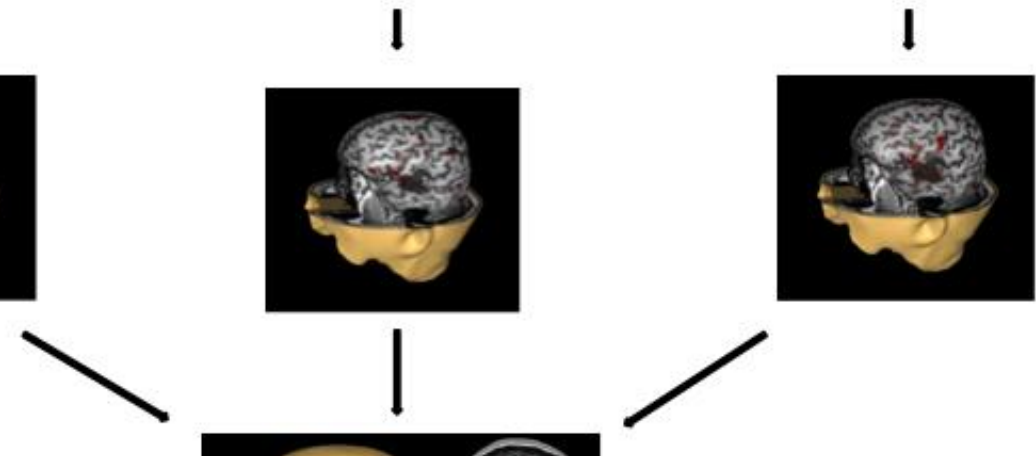

Figure 1. Individualized stimulation site was determined based on overlapping BOLD signals in the left hemisphere across three speech-language tasks. In each image, orange markers were placed using the neuronavigation system to identify locations of cortical activation. fMRI BOLD signals for each task were overlaid onto 2D images (A) and a 3D head reconstruction (B). While it is impossible to encompass all areas of activation, care was taken to locate stimulation sites with demonstrated activation in at least two of the three tasks. Lastly, scalp projections (C) of each stimulation site were generated and verified to fit underneath the surface area of the electrode.

Table 1. Demographics, clinical characteristics, tDCS allocation, and stimulation site for study participants.

\begin{tabular}{|c|c|c|c|c|c|c|c|c|c|c|c|c|c|c|}
\hline Participant & $\begin{array}{c}\text { Age } \\
\text { (Years) }\end{array}$ & Sex & $\begin{array}{c}\text { Lesion } \\
\text { Size } \\
(\mathrm{mL})\end{array}$ & $\begin{array}{c}\text { TPO } \\
\text { (Months) }\end{array}$ & $\begin{array}{c}\text { Educ } \\
\text { (Years) }\end{array}$ & AQ & LQ & $\mathrm{CQ}$ & SS & Comp & Rpt & Nmg & $\begin{array}{c}\text { Polarity vs. } \\
\text { Sham }\end{array}$ & $\begin{array}{c}\text { Stimulation } \\
\text { Site }\end{array}$ \\
\hline \multicolumn{15}{|c|}{ WAB-R AQ > 55} \\
\hline 1 JONRA & 46.1 & $\mathrm{M}$ & 66.9 & 6.3 & 16 & 70.3 & 64.0 & 70.9 & 12 & 8.0 & 6.6 & 8.6 & A-tDCS & MFG \\
\hline 2 SHAER & 51.5 & $\mathrm{M}$ & 80.3 & 29.2 & 16 & 61.3 & 61.9 & 69.7 & 10 & 9.1 & 4.7 & 6.9 & A-tDCS & $\mathrm{FP}$ \\
\hline 3 LEELO & 57.1 & $\mathrm{~F}$ & 92.8 & 38.9 & 17 & 74.3 & 74.8 & 76.2 & 14 & 8.8 & 7.4 & 7.0 & C-tDCS & $\mathrm{FP}$ \\
\hline 4 TONMA & 57.8 & $\mathrm{~F}$ & 94.7 & 50.9 & 16 & 63.9 & 71.1 & 73.5 & 12 & 9.4 & 5.7 & 4.9 & C-tDCS & M1 \\
\hline 5 PEACA & 55.3 & $\mathrm{~F}$ & 76.6 & 155.7 & 12 & 70.1 & 65.1 & 69.8 & 12 & 8.7 & 7.3 & 7.1 & S-tDCS & MFG \\
\hline 6 PIWTO & 61.4 & $\mathrm{M}$ & 155.8 & 53.3 & 13 & 75.3 & 65.8 & 70.3 & 13 & 8.1 & 9.5 & 7.1 & S-tDCS & SMG \\
\hline \multicolumn{15}{|c|}{ WAB-R AQ < 55} \\
\hline 7 ANDJA & 46.1 & $\mathrm{M}$ & 118.6 & 35.6 & 12 & 45.2 & 46.4 & 54.2 & 8 & 5.5 & 4.8 & 4.3 & A-tDCS & MFG \\
\hline 8 HOWSH & 55.7 & $\mathrm{~F}$ & 136.4 & 9.2 & 17 & 47.1 & 37.4 & 42.2 & 9 & 6.1 & 5.6 & 2.9 & A-tDCS & MFG \\
\hline 9 KARYA & 58.8 & $\mathrm{M}$ & 46.5 & 6.2 & 16 & 38.7 & 37.6 & 45.8 & 9 & 5.2 & 4.1 & 1.1 & C-tDCS & IFG \\
\hline 10 TRAWI & 64.9 & $\mathrm{M}$ & 83.3 & 18.6 & 18 & 44.0 & 58.0 & 61.6 & 7 & 8.3 & 2.8 & 3.9 & C-tDCS & IFG \\
\hline $11 \mathrm{KANJO}$ & 71.1 & $\mathrm{M}$ & 24.5 & 6.2 & 16 & 54.3 & 60.3 & 65.6 & 7 & 7.6 & 4.8 & 7.8 & S-tDCS & MFG \\
\hline 12 BUTFR & 54.7 & $\mathrm{M}$ & 56.1 & 7.3 & 18 & 22.3 & 30.0 & 38.4 & 4 & 4.8 & 1.9 & 0.5 & S-tDCS & M1 \\
\hline
\end{tabular}

Note. $\mathrm{TPO}=$ Time Post Onset, Educ = Education, WAB-R = Western Aphasia Battery-Revised [25], AQ = Aphasia Quotient, LQ = Language Quotient, $\mathrm{CQ}=$ Cortical Quotient, $\mathrm{SS}=$ Spontaneous Speech Score (maximum $=20)$, Comp = Auditory Verbal Comprehension (maximum $10), \mathrm{Rpt}=$ Repetition $($ maximum $=10)$, and Nmg = Naming and Word Finding (maximum $=10)$. A-tDCS $=$ anodal, $C-\mathrm{tDCS}=\mathrm{cathodal}$, and S-tDCS = sham. IFG = inferior frontal gyrus, MFG = middle frontal gyrus, $\mathrm{FP}=$ frontal pole, $\mathrm{M} 1=$ precentral gyrus, and $\mathrm{SMG}=$ supramarginal gyrus. 

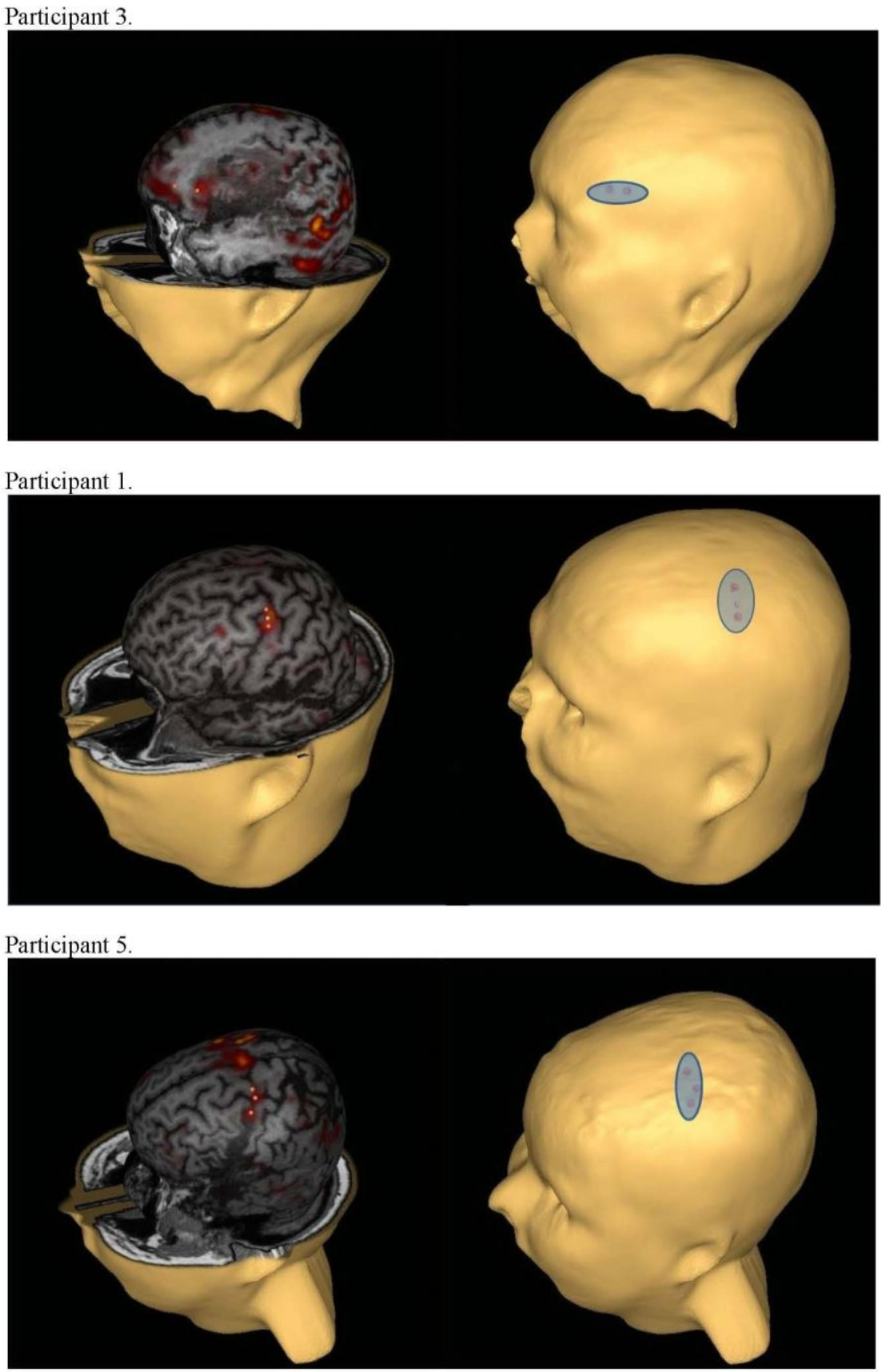

Figure 2. Examples of fMRI-navigated, perilesional stimulation sites across three participants. The figure illustrates how the fMRI "hot spots" were incorporated into one electrode location with the proper orientation. For Participant 3, the electrode placement was based on activation in tasks 2 and 3. For Participant 1, the electrode site incorporated activity on all three fMRI tasks. For Participant 5, electrode placement was specific to tasks 1 and 2. Thus, electrode placement was individualized for each participant. The reference electrode was placed horizontally over the right contralateral supraorbital area for all participants. 


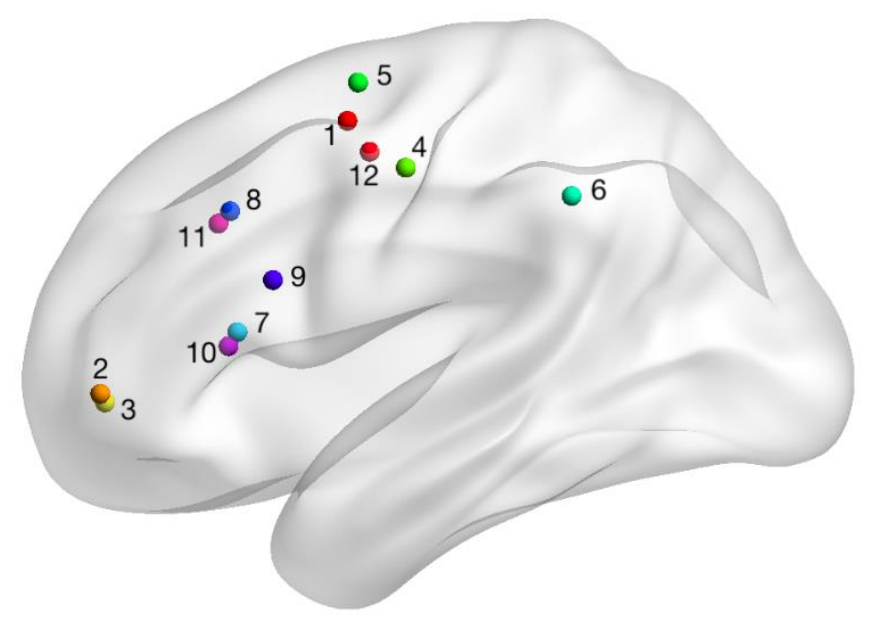

Figure 3. Plotted fMRI-navigated stimulation sites for all study participants on a model of a standard left hemisphere. Numbers correspond to the participant number (Table 1).

\subsection{Randomization and Blinding}

Participants were randomized to one of three treatment arms (A-tDCS, C-tDCS, or S-tDCS); each arm was delivered simultaneously with computerized SLT. Participants were randomized after all baseline assessments were completed and the stimulation site had been determined. Randomization was generated in advance by a statistician who provided the principal investigator (LRC) with individual, sequentially numbered sealed envelopes containing the randomization for prospective participants. Randomization was blocked for every three eligible participants and stratified by aphasia severity (i.e., a cut-off score of 55 on the WAB-R AQ). The principal investigator, who was not involved in recruitment, assessment, or treatment, then allocated the envelopes. Participants, care providers/family members, and the evaluator remained blinded to group assignment throughout the study. In the sham treatment arm, stimulation was provided in a programmed, ramp-like fashion for $30 \mathrm{~s}$, and then shut off. The parameters for the sham stimulation were chosen based on published reports that perceived that sensations from tDCS (e.g., tingling on the skin) are transient and fade after the first $30 \mathrm{~s}$ of stimulation [30]. Therefore, participants who received sham $\mathrm{tDCS}$ perceived physical sensations of tingling that were similar to the sensations perceived by participants who received tDCS for $13 \mathrm{~min}$. To confirm proper blinding of sham versus active tDCS, following completion of the post-treatment assessment, participants were asked to guess whether they had received active or sham stimulation, even though treatment allocation remained undisclosed. The four participants who received sham tDCS all indicated that they thought they had received active stimulation. Of the eight participants who received active stimulation, only one participant (\#2) indicated that he thought he had received sham stimulation.

\section{4. tDCS Intervention}

tDCS was delivered using a constant current stimulator (Dupel Iontophoreis System, Empi, MN) via an $8 \mathrm{~cm}^{2}$ oblong $(2 \times 4)$ saline-soaked sponge electrode placed directly over the established stimulation site (Dupel BLUE, MN). A self-adhesive carbonized reference anode $\left(48 \mathrm{~cm}^{2}\right)$ was placed on the forehead, directly above the contralesional orbit.

Treatment sessions lasted $90 \mathrm{~min}$ and were completed five days a week for six weeks, for a total of 30 sessions. Across all study arms, $1 \mathrm{~mA}$ of active (anodal or cathodal) or sham tDCS was applied to the lesioned left hemisphere during the first $13 \mathrm{~min}$ of the 90-minute speech-language treatment session. The parameters for active tDCS were strategically chosen to induce and prolong current-driven increases in cortical excitability, lasting up to $90 \mathrm{~min}$ post-stimulation [14-16]. Nitshe and colleagues have demonstrated that after short, 5- or 7-minute applications of low-intensity A-tDCS or C-tDCS (1mA) to the upper limb motor cortex, the amplitude of motor-evoked potentials (MEPs) quickly returned 
to baseline within a few min after stimulation had ended [14-16]. However, 13 min of A-tDCS at the same low-level intensity produced extended periods of elevated MEPs (i.e., approximately $150 \%$ of baseline), which were well-maintained up to 90 min poststimulation [15]. Careful consideration of tolerability and safety was also reflected in the decision to apply a current intensity of $1 \mathrm{~mA}$ across the longer duration of 13 min in the present study [31,32].

The stimulation was initially increased in a programmed, ramp-like fashion over several seconds until reaching $1 \mathrm{~mA}$ for all participants. Within both A-tDCS and C-tDCS arms, the stimulation was then maintained for a total of $13 \mathrm{~min}$; however, in the sham condition, the stimulation was turned off after $30 \mathrm{~s}$, outside of the view of the participant to maintain blinding. Additionally, a timer was set for $15 \mathrm{~min}$ after the start of the SLT, at which time the electrode and tDCS leads were removed for all participants prior to completing the remaining 75 min of computerized SLT.

\subsection{Concurrent Language Intervention}

Concurrent SLT intervention consisted of two, evidence-based treatment protocols that provide practice in auditory and reading comprehension of sentences and short scripts as well as production of functional language. Both treatments were administered via computer with a "virtual therapist" to ensure treatment fidelity across sessions and participants. Having two treatments served to increase participant engagement by breaking up any monotony that might occur during the lengthy 90 -minute treatment session. First, participants received two sequential 15-minute intervals of Oral Reading for Language in Aphasia $\left(\right.$ ORLA $\left.^{\circledR}\right)$. The first 15 min of ORLA ${ }^{\circledR}$ was always paired with the tDCS. With ORLA ${ }^{\circledR}$, the participant repeatedly practices reading aloud sentences, first in unison with the "virtual therapist" and then independently [33-35]. Participants with WAB-R AQ scores less than 55 practiced sentences that were 3-5 words long, whereas those with WAB-R AQ scores greater than 55 practiced sentences that were 8-10 words long. The sentence stimuli for each ORLA ${ }^{\circledR}$ treatment session were randomly selected by the computer from a pool of 150 sentences that were available at each level (i.e., 3-5 word level and 8-10 word level). Following a five-minute rest break, the participant then completed a 30-minute interval of AphasiaScripts ${ }^{\circledR}$, which also incorporates reading aloud of sentences, but now embedded within scripted conversations [36-38]. Two sets of six scripts each were available, one set of shorter scripts with grammatically simple short sentences and another set of longer scripts with more complex sentences. Similar to ORLA ${ }^{\circledR}$, the participant's WAB-R AQ was used to determine which set of scripts was selected since overall script complexity of each set was similar to the complexity of the two levels of ORLA ${ }^{\circledR}$. However, sentence stimuli practiced during AphasiaScripts ${ }^{\circledR}$ were different from those practiced during ORLA ${ }^{\circledR}$. Each script was practiced for five days, with a new script introduced at the beginning of each week of treatment. The last 30-min interval of treatment was continued reading aloud practice via ORLA ${ }^{\circledR}$. Sample screenshots of AphasiaScripts ${ }^{\circledR}$ and ORLA ${ }^{\circledR}$ are included in Figure 4. A schematic of the procedures within a single, daily treatment session are illustrated in Figure 5. Participants did not receive any other individual or group aphasia treatment during their participation in the study, including the six-week follow-up period. 
(A)

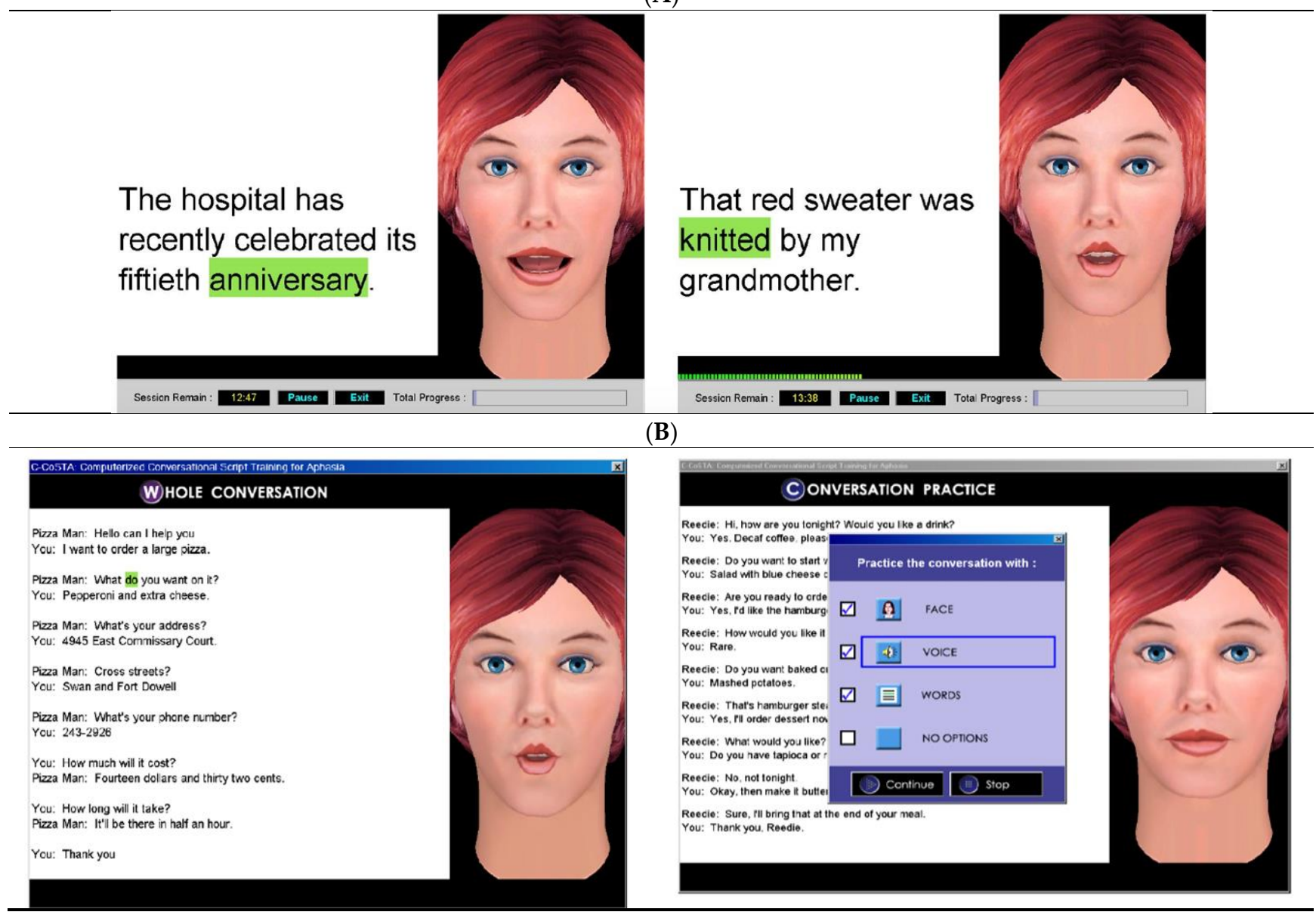

Figure 4. Sample screenshots showing the "virtual therapist" and examples of sentence and script stimuli in (A) AphasiaScripts ${ }^{\circledR}$ and (B) ORLA ${ }^{\circledR}$. Words are highlighted in green as the virtual therapist reads the sentences aloud by herself and then in unison with the participant. When the participant reads the sentences aloud in unison and independently, the green highlighting of the words and the virtual therapist's mouth movements provide cues that help with the accuracy and rate of production of the words and sentences. In AphasiaScripts ${ }^{\circledR}$, these cues can be removed one at a time during the conversation practice.

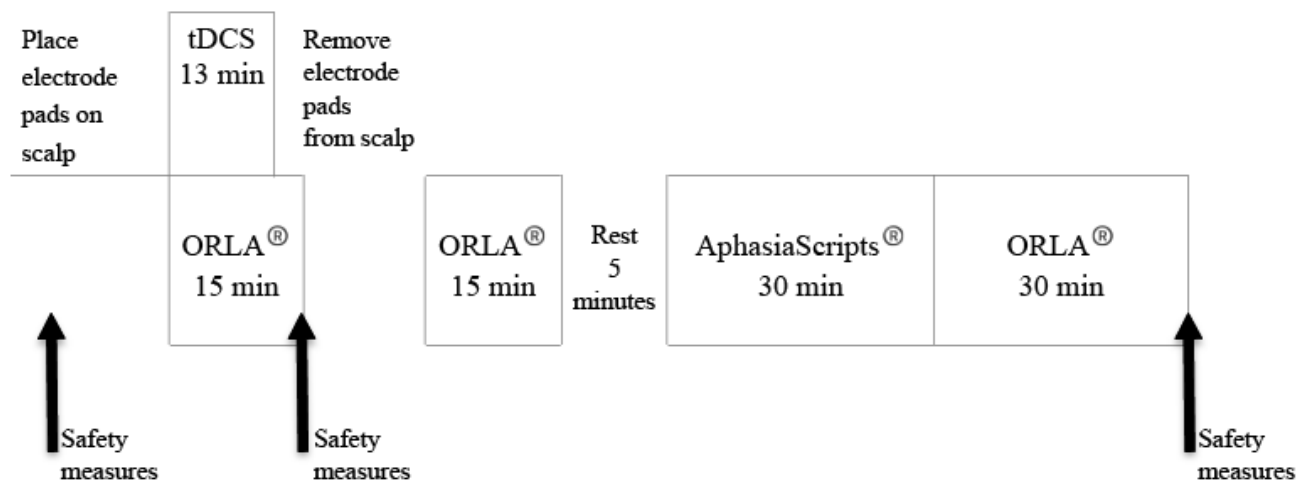

Figure 5. Schematic of a daily treatment session showing the sequence and length of time of each of the behavioral treatments: Oral Reading for Language in Aphasia $\left(\right.$ ORLA $\left.^{\circledR}\right)$ and AphasiaScripts ${ }^{\circledR}$. The tDCS was always applied with ORLA $^{\circledR}$. The schematic also shows when safety measures were collected. 


\subsection{Outcome Measures}

Outcome measures of safety and tolerability included vital signs of temperature, heart rate, and blood pressure. Vitals were completed using digital sensors at three time points during each session: before and after the tDCS interval and the end of the 90-minute treatment session. In addition, self-reported side effects were obtained at the same three time points using an aphasia-friendly questionnaire with an attached 10-point scale (where 10 represents the highest degree of discomfort). The same questionnaire was used during weekly follow-up phone calls to the participants throughout a six-week maintenance phase to document and address any long-term side effects from the tDCS.

The primary language outcome measure was the AQ of the WAB-R which has been identified as a core outcome measurement instrument for aphasia to be used in Phase I-IV clinical trials $[25,39]$. The Language Quotient (LQ) of the WAB-R which encompasses additional reading comprehension and written expression skills and the Communication Effectiveness Index (CETI) [40] served as secondary language outcomes. The CETI measures functional communication skills as reported by a caregiver. Caregivers were blinded to treatment allocation as well as patient performance during assessment and scoring of language outcome measures. The aforementioned language measures were administered before and after the six weeks of treatment and at six weeks following the end of treatment (i.e., follow-up). A gain of 5 points or greater on the WAB-R AQ and LQ was considered to be clinically significant, whereas a gain of 10 points or greater was considered to be clinically significant on the CETI $[40,41]$.

Behavioral probe measures of oral reading accuracy and rate (words per minute, wpm) were also obtained at each assessment (pre-treatment, post-treatment, and followup) as well as weekly during the treatment interval. Probe tasks, including baseline probes, required the participant to read aloud ten sentences, randomly selected by the computer program from the pool of 150 trained ORLA ${ }^{\circledR}$ stimuli. The participant's sentence productions were scored and rated for percent accuracy and words per minute (wpm) according to the Naming and Oral Reading for Language in Aphasia 6-point scale (NORLA6) [42]. Fifteen percent of all probes were randomly selected and checked for point-to-point inter-rater reliability of scoring between the treating clinician and an independent evaluator not associated with the study. Inter-rater reliability was $96.9 \%$ for accuracy and $97.4 \%$ for rate.

Neurophysiological changes were quantified through the number of activated voxels within a $5 \mathrm{~mm}$ area around the lesion (perilesional cortex) and the ratio of activated voxels between the entire left vs. right hemisphere (left/right ratio) for each fMRI task and compared between pre- and post-treatment.

No changes were made to the outcomes after the trial commenced.

\subsection{Analyses}

For the primary language outcome measure, the WAB-R AQ, inferential analyses were conducted in SPSS 24.0 with post-hoc power analyses conducted with $\mathrm{G}^{*}$ Power 3.1.9.7. Significance level was set at $p<0.05$. A one-way ANOVA explored significant differences among the groups in baseline WAB-R AQ performance. Paired $t$-tests explored significant post-treatment changes in WAB-R AQ scores within each group. A mixed-model, two-way ANOVA was conducted to explore changes in WAB-R AQ at post-treatment by treatment group. Effect sizes for ANOVA and $t$-test results were calculated using (partial) eta squared ( 12$)$ and Cohen's $d$, respectively, and interpreted based on Cohen (1988). As an exploratory pilot study with small sample size, there is risk for not detecting a true difference in outcomes across treatments due to lack of power. Thus, a post-hoc power analysis based on the observed difference among WAB-R AQ group means was conducted to estimate the sample sizes necessary for future studies to detect statistical differences among these treatment arms. 
Group means and individual change scores on the standardized language measures (WAB-R AQ, WAB-R LQ, and the CETI) were also descriptively analyzed in relation to established benchmarks of clinical significance $[40,41,43]$.

NORLA-6 performance varied greatly across participants at baseline. Therefore, NORLA-6 performance from (1) pre- to post-treatment and (2) pre-treatment to follow-up was calculated as a percent of gain for both individual participants and by group. Percent gain from pre- to post-treatment was calculated as the mean of three post-treatment probe scores minus the mean of three pre-treatment probe scores divided by the mean of the three pre-treatment probe scores. The percent gain from pre-treatment to follow-up testing was calculated similarly as the mean of the two follow-up probe scores minus the mean of the three pre-treatment probe scores divided by the mean of the three pre-treatment probes scores. Effect sizes for NORLA- 6 gains by individual participants were calculated using Busk and Serlin's $d$ statistic with reference to established benchmarks for a small (2.6), medium (3.9), or large (5.8) effect size that were derived from meta-analyses of aphasia treatments [44-46]. A priori determination of a clinically significant effect size was greater than 3.9 (medium effect).

In regard to exploring changes in cortical activation patterns, the duration of each fMRI task varied slightly with 284 volumes collected during Task 1, 230 volumes during Task 2, and 285 volumes during Task 3 . The first six volumes of each run were discarded to allow the MRI signal to reach equilibrium. SPM8 (http:/ / www.fil.ion.ucl.ac.uk/spm/ software/spm8/) was used to analyze images. Functional images were realigned with the first volume, re-sliced, co-registered to the T1 anatomical image, spatially smoothed with a $6 \mathrm{~mm}$ full-width at half-maximum Gaussian kernel, and high-pass filtered (cutoff period of $256 \mathrm{~s}$ for Tasks 1 and 2 and $128 \mathrm{~s}$ for Task 3 . Fixed effect analyses were conducted on each task using a generalized linear model. Each acquisition block was modeled independently and convolved with the canonical hemodynamic response function combined with time and dispersion derivatives. Individual activation maps (F-contrast) for Tasks 1 and 3 were contrasted between baseline and task. For Task 2, active maps were contrasted between overt and covert conditions. The number of and ratio of activated voxels was counted for both left and right hemispheres using the functional Magnetic Resonance Imaging of the Brain Software Library (FSL) image analysis suite's FSLSTATS program (http:/ /www.fmrib.ox.ac.uk/fsl/) [47]. The manually segmented lesion masks based on anatomical images were spatially transformed to the individuals' functional space using the FLIRT toolbox (part of FSL) [48-50]. Perilesional masks were created by dilating the lesion masks using a $4 \mathrm{~mm}$ Gaussian kernel. Activated voxels within the perilesional masks on the left hemisphere were obtained to indicate the level of perilesional brain activation.

\section{Results}

Twenty participants were screened with 12 participants enrolled in the study. A CONSORT flow diagram is included as Figure 6; Table 1 reports demographics and stimulation site for enrolled participants. Eight participants were excluded as follows: three participants had WAB-R AQ scores outside of target range, two participants did not demonstrate cortical activation in the left hemisphere across the fRMI tasks, two participants exhibited MRI/tDCS contraindications, and one participant exhibited fluent aphasia during pretreatment assessment. We note that Participant 12 was enrolled even though he scored slightly below the WAB-R inclusion cut-off score of 25 because the study support was ending; this was approved by the IRB. 
CONSORT 2010 Flow Diagram

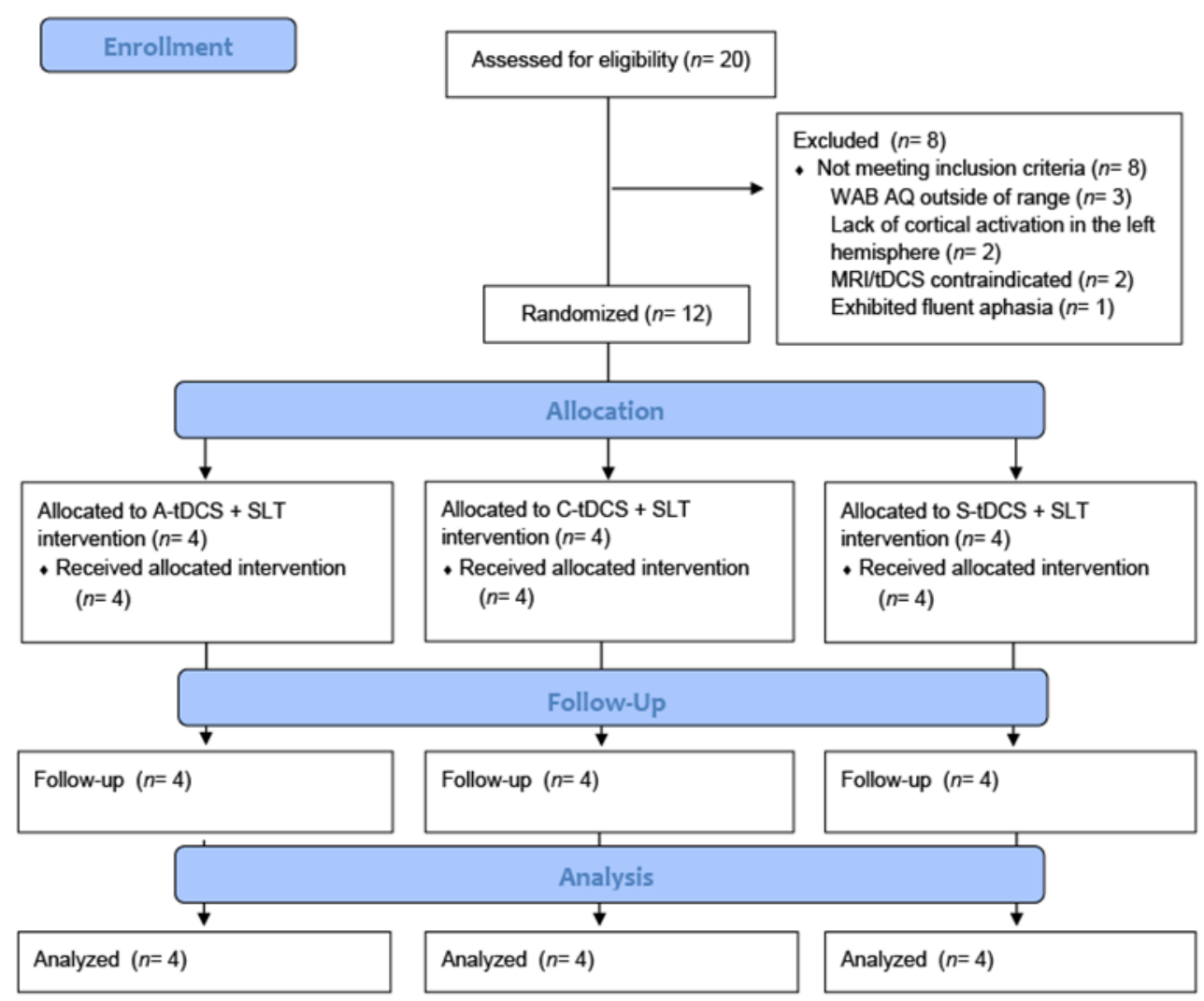

Figure 6. CONSORT 2010 Flow Diagram. Note. A-tDCS = anodal, C-tDCS = cathodal, and S-tDCS = sham stimulation. WAB-R AQ = Western Aphasia Battery-Revised Aphasia Quotient.

All participants completed the 30 treatment sessions including the monitoring of vital signs and adverse events. All participants completed weekly follow-up phone calls during the six-week follow-up interval after the conclusion of treatment. Participants also completed primary and secondary measures of language outcomes, except for Participant 8 , who did not have a significant other available to complete the CETI and, therefore, CETI scores are missing. In addition, during the follow-up assessment, Participant 8 declined to continue with follow-up testing after the administration of the WAB-R AQ; thus, the remaining follow-up data points are missing.

\subsection{Safety and Tolerability of Prolonged tDCS}

Vitals were stable and remained within normal limits during the six weeks of treatment for all participants. No serious adverse events were reported across the treatment interval, in weekly phone calls between the end of treatment and the six-week follow-up assessment, or at follow-up appointments. Table 2 shows the frequency of side effects (tingling/itching under the electrodes, fatigue, headache, dizziness and dry mouth) that were reported during the treatment sessions. It also shows the degree of discomfort experienced and the weeks during the treatment period that these side effects occurred. With few exceptions, degree of discomfort was usually rated at 3 or less on a 10-point scale where 0 indicated no discomfort and 10 indicated maximum discomfort. Six participants, including two participants who received sham tDCS, reported increasing tingling and itchiness under the electrode during the initial weeks of the treatment. For one participant (\#8), the tingling occurred in more than half of the treatment sessions, reportedly increasing in frequency and magnitude during weeks four, five and six, and extending down her right arm to 
her fingers; however, overall the degree of discomfort averaged a 3 on the 10-point scale. Fatigue was another side effect that several participants reported, but it is not clear whether these reports were related to the tDCS or other factors. For example, Participant 1 reported excessive tiredness during weeks four to six, which was rated a 2 or 3 on the 10-point scale and coincided with the start of a new blood pressure medication (i.e., Lisinopril) for which excessive tiredness is a noted side effect.

Table 2. Reported side effects for each participant.

\begin{tabular}{|c|c|c|c|c|c|c|c|c|c|c|c|c|c|c|c|c|}
\hline \multirow[b]{3}{*}{ ID } & \multirow[b]{3}{*}{ Arm * } & \multicolumn{15}{|c|}{ Side Effect } \\
\hline & & \multicolumn{3}{|c|}{ Tingling/Itching } & \multicolumn{3}{|c|}{ Fatigue } & \multicolumn{3}{|c|}{ Headache } & \multicolumn{3}{|c|}{ Dizzy } & \multicolumn{3}{|c|}{ Dry Mouth } \\
\hline & & $\begin{array}{l}\text { Number } \\
\text { of } \\
\text { Sessions }{ }^{1}\end{array}$ & $\underset{2}{\mathrm{Avg}}$ & Week $^{3}$ & $\begin{array}{l}\text { Number } \\
\text { of } \\
\text { Sessions }\end{array}$ & Avg & Week & $\begin{array}{l}\text { Number } \\
\text { of } \\
\text { Ses- } \\
\text { sions }\end{array}$ & Avg & Week & $\begin{array}{l}\text { Number } \\
\text { of } \\
\text { Sessions }\end{array}$ & Avg & Week & $\begin{array}{l}\text { Number } \\
\text { of } \\
\text { Sessions }\end{array}$ & Avg & Week \\
\hline 1 & A & 6 & 2.2 & $1-2$ & 6 & 2.9 & $4-6$ & - & - & - & - & - & - & 1 & 1.0 & 1 \\
\hline 2 & A & 3 & 5.3 & 1 & 7 & 3.0 & $3-5$ & - & - & - & 1 & 1.0 & 3 & - & - & - \\
\hline 3 & C & - & - & - & - & - & - & - & - & - & - & - & - & - & - & - \\
\hline 4 & $\mathrm{C}$ & - & - & - & - & - & - & - & - & - & - & - & - & - & - & - \\
\hline 5 & $\mathrm{~S}$ & - & - & - & - & - & - & - & - & - & - & - & - & - & - & - \\
\hline 6 & $S$ & - & - & - & - & - & - & - & - & - & - & - & - & - & - & - \\
\hline 7 & $\mathrm{~A}$ & - & - & - & 10 & 4.1 & $2-6$ & - & - & - & 1 & 2.0 & 1 & - & - & - \\
\hline 8 & A & 24 & 3.0 & $1-6$ & - & - & - & 1 & 1.5 & 4 & - & - & - & - & - & - \\
\hline 9 & C & 13 & 4.6 & $1-5$ & 5 & 2.0 & $1,3,4$ & 1 & 2.0 & 3 & 13 & 2.0 & $2-6$ & - & - & - \\
\hline 10 & C & - & - & - & 3 & 1.9 & $1,2,4$ & 2 & 1.5 & 1,4 & - & - & - & - & - & - \\
\hline 11 & $S$ & 6 & 1.0 & $1-3$ & 2 & 6.0 & 1,6 & 1 & 2.0 & 1 & - & - & - & 2 & 1.0 & 1,3 \\
\hline 12 & S & 14 & 3.4 & $1-3$ & - & - & - & - & - & - & - & - & - & - & - & - \\
\hline
\end{tabular}

${ }^{*}$ Arm $=$ Subjects randomized to Anodal (A), Cathodal (C), or Sham (S) tDCS. ${ }^{1}$ Number of sessions in which side effect was reported out of a total of 30 treatment sessions; ${ }^{2}$ Average rating on scale of $0-10$ with $0=$ no discomfort and $10=$ maximum discomfort; ${ }^{3}$ Weeks in which side effect was reported.

\subsection{Language Outcome Measures}

Gains in standardized language outcome measures are first reported by group (Table 3) and subsequently for individuals (Table 4). Mean percent gains in oral reading accuracy and rate are reported by group in Table 5 . Individual gains in oral reading performance at post-treatment and follow-up are reported in Table 6.

Table 3. Mean (Std Dev) gains in language performance on the Western Aphasia Battery-Revised (WAB-R) and caregiver-reported communication effectiveness by group at both post-treatment and six-week follow-up.

\begin{tabular}{cccccccc}
\hline Group & $\begin{array}{c}\text { Number of } \\
\text { Participants }\end{array}$ & $\begin{array}{c}\text { AQ } \\
\text { Pre-Post }\end{array}$ & $\begin{array}{c}\text { AQ } \\
\text { Pre-F }\end{array}$ & $\begin{array}{c}\text { LQ } \\
\text { Pre-Post }\end{array}$ & $\begin{array}{c}\text { LQ } \\
\text { Pre-F }\end{array}$ & $\begin{array}{c}\text { CETI } \\
\text { Pre-Post }\end{array}$ & $\begin{array}{c}\text { CETI } \\
\text { Pre-F }\end{array}$ \\
\hline A-tDCS & 4 & $5.2(1.7)$ & $7.4(2.2)$ & $5.7(1.8)$ & $6.6(1.4)^{1}$ & $13.9(13.3)^{1}$ & $14.5(17.6)^{1}$ \\
C-tDCS & 4 & $5.4(2.5)$ & $5.7(5.6)$ & $3.7(2.6)$ & $6.2(2.6)$ & $17.2(2.3)$ & $20.8(2.1)$ \\
S-tDCS & 4 & $4.8(7.7)$ & $3.1(8.4)$ & $4.6(2.2)$ & $3.3(4.0)$ & $7.2(6.3)$ & $10.2(8.0)$ \\
\hline
\end{tabular}

${ }^{1}$ Descriptive statistics were calculated only on three participants Note. Western Aphasia Battery-Revised (WABR) $[25] \mathrm{AQ}=$ Aphasia Quotient and $\mathrm{LQ}=$ Language Quotient. CETI $=$ Communication Effectiveness Index as rated by a caregiver [38]. Pre-Post $=$ gain from pre-treatment to post-treatment, and Pre-F $=$ gain from pre-treatment to follow-up. NA = not available. A-tDCS = anodal, $\mathrm{C}-\mathrm{tDCS}=$ cathodal, and S-tDCS $=$ sham.

\subsubsection{Primary Outcome Measure (WAB-R AQ)}

The three groups did not significantly differ in WAB-R AQ scores at baseline, $F(2,9)=0.002, p=0.998$. Overall WAB-R AQ scores demonstrated significant gains at post-treatment, $F(1,9)=13.987, p=0.005, \eta 2=0.608$; but there was no significant difference in group, $F(1,9)=0.002, p=0.998, \eta 2=0.000$, nor an interaction effect $(p=0.985, \eta 2=0.003)$. Significant gains in WAB-R AQ scores were demonstrated for the anodal $t(3)=6.107$, $p=0.009, d=3.06$; cathodal groups $t(3)=4.416, p=0.022, d=2.21$ with large effects sizes, but not for the sham group $t(3)=1.255, p=0.298, d=0.63$.

Post-hoc power analysis was based on present results, presuming a one-way independent ANOVA given a significance level of $p=0.05$ and equal treatment allocation, which indicated that 33 participants would be required for a lower powered study $(\beta=0.2)$ and 84 participants would be required for a higher $(\beta=0.5)$ powered study. 
Both the A-tDCS and C-tDCS groups demonstrated a clinically significant improvement (mean gain $\geq 5$ points) on the $A Q$ from pre- to post-treatment ( 5.2 and 5.4 , respectively) and from pre-treatment to follow-up (7.4 and 5.7, respectively; Table 3). The mean gains of the S-tDCS group did not meet criteria for clinically significant gains on the AQ for either time interval. At an individual level (Table 4), all four participants receiving A-tDCS, three of four participants receiving C-tDCS, and only one participant receiving S-tDCS stimulation demonstrated clinically significant changes on the $A Q$ from pre-treatment to post-treatment and/or follow-up testing.

Table 4. Individual scores and gains in language performance per the WAB-R (AQ and LQ) and caregiver-reported gains on the CETI.

\begin{tabular}{|c|c|c|c|c|c|c|c|c|c|c|c|c|c|c|c|c|}
\hline Participant & $\begin{array}{c}\text { Polarity } \\
\text { vs. } \\
\text { Sham }\end{array}$ & $\begin{array}{l}\text { AQ } \\
\text { Pre }\end{array}$ & $\begin{array}{l}\text { AQ } \\
\text { Post }\end{array}$ & $\underset{\mathrm{F}}{\mathrm{AQ}}$ & $\begin{array}{l}\text { AQ } \\
\text { Pre- } \\
\text { Post }\end{array}$ & $\begin{array}{c}\text { AQ } \\
\text { Pre- } \\
\text { F }\end{array}$ & $\begin{array}{l}\text { LQ } \\
\text { Pre }\end{array}$ & $\begin{array}{l}\text { LQ } \\
\text { Post }\end{array}$ & $\underset{\mathrm{F}}{\mathrm{LQ}}$ & $\begin{array}{l}\text { LQ } \\
\text { Pre- } \\
\text { Post }\end{array}$ & $\begin{array}{c}\text { LQ } \\
\text { Pre-F }\end{array}$ & $\begin{array}{c}\text { CETI } \\
\text { Pre }\end{array}$ & $\begin{array}{l}\text { CETI } \\
\text { Post }\end{array}$ & $\begin{array}{c}\text { CETI } \\
\text { F }\end{array}$ & $\begin{array}{l}\text { CETI } \\
\text { Pre- } \\
\text { Post }\end{array}$ & $\begin{array}{l}\text { CETI } \\
\text { Pre-F }\end{array}$ \\
\hline \multicolumn{17}{|c|}{ WAB-R AQ > 55} \\
\hline 1 & A-tDCS & 70.3 & 76.6 & 77.1 & 6.3 & 6.8 & 64.0 & 67.8 & 69.7 & 3.8 & 5.7 & 47.6 & 76.9 & 82.3 & 29.3 & 34.7 \\
\hline 2 & A-tDCS & 61.3 & 68.3 & 71.7 & 7.0 & 10.4 & 61.9 & 66.4 & 70.1 & 4.5 & 8.2 & 33.3 & 38.9 & 39.8 & 5.7 & 6.6 \\
\hline 3 & C-tDCS & 74.3 & 77.7 & 75.5 & 3.4 & 1.2 & 74.8 & 77.4 & 79.6 & 2.6 & 4.8 & 45.9 & 60.2 & 65.8 & 14.3 & 19.9 \\
\hline 4 & C-tDCS & 63.9 & 67.6 & 71.5 & 3.7 & 7.6 & 71.1 & 72.1 & 74.4 & 1.0 & 3.3 & 54.8 & 71.3 & 75.1 & 16.4 & 20.3 \\
\hline 5 & S-tDCS & 70.1 & 72.7 & 69.5 & 2.6 & -0.6 & 65.1 & 67.9 & 63.2 & 2.8 & -1.9 & 53.8 & 54.9 & 55.9 & 1.1 & 2.1 \\
\hline 6 & S-tDCS & 75.3 & 73.7 & 71.4 & -1.6 & -3.9 & 65.8 & 69.7 & 68.1 & 3.9 & 2.3 & 47.6 & 50.6 & 54.3 & 3.0 & 6.8 \\
\hline \multicolumn{17}{|c|}{ WAB-R AQ $<55$} \\
\hline 7 & A-tDCS & 45.2 & 49.1 & 52.2 & 3.9 & 7.0 & 46.4 & 53.4 & 52.2 & 7.0 & 5.8 & 70.1 & 76.9 & 72.4 & 6.9 & 2.3 \\
\hline 8 & A-tDCS & 47.1 & 50.7 & 52.4 & 3.6 & 5.3 & 37.4 & 44.8 & NA & 7.4 & NA & NA & NA & NA & NA & NA \\
\hline 9 & C-tDCS & 38.7 & 44.5 & 39.8 & 5.8 & 1.1 & 37.6 & 44.4 & 46.6 & 6.8 & 9.0 & 70.4 & 88.8 & 94.3 & 18.3 & 23.9 \\
\hline 10 & C-tDCS & 44.0 & 52.7 & 56.8 & 8.7 & 12.8 & 58.0 & 62.4 & 65.8 & 4.4 & 7.8 & 44.2 & 63.8 & 63.4 & 19.6 & 19.2 \\
\hline 11 & S-tDCS & 54.3 & 70.3 & 69.6 & 16 & 15.3 & 60.3 & 68.1 & 66.8 & 7.8 & 6.5 & 24.7 & 39.1 & 45.7 & 14.4 & 21.0 \\
\hline 12 & S-tDCS & 22.3 & 24.6 & 24.4 & 2.3 & 1.9 & 30.0 & 33.9 & 36.3 & 3.9 & 6.3 & 38.2 & 48.7 & 48.4 & 10.5 & 10.2 \\
\hline
\end{tabular}

Note. Western Aphasia Battery-Revised (WAB) [25] AQ = Aphasia Quotient and LQ = Language Quotient. CETI = Communication Effectiveness Index as rated by a caregiver [38] Pre $=$ Pre-treatment. Score, Post $=$ post-treatment score, $\mathrm{F}=$ follow-up score, Pre-Post $=$ gain from pre-treatment to post-treatment, and Pre-F = gain from pre-treatment to follow-up. NA = not available. A-tDCS $=$ anodal, $C-$ tDCS $=$ cathodal, and S-tDCS $=$ sham .

Table 5. Mean percent gains (Std Dev) in oral reading accuracy and rate by group at both post-treatment and six-week follow-up testing.

\begin{tabular}{cccccc}
\hline Group & $\begin{array}{c}\text { Number of } \\
\text { Partici- } \\
\text { pants }\end{array}$ & $\begin{array}{c}\text { Oral Reading Accuracy } \\
\text { Gain (SD) } \\
\text { at Post- } \\
\text { Treatment }\end{array}$ & $\begin{array}{c}\text { Oral Reading Accuracy } \\
\text { Gain (SD) } \\
\text { at Follow-Up Testing }\end{array}$ & $\begin{array}{c}\text { Oral Reading } \\
\text { Rate Gain (SD) } \\
\text { at Post-Treatment }\end{array}$ & $\begin{array}{c}\text { Oral Reading Rate } \\
\text { Gain (SD) }\end{array}$ \\
\hline A-tDCS & 4 & $47.3(34.7)$ & $25.7(0.6)$ & $63.2(62.8)$ & $120.7(74.3))^{1}$ \\
C-tDCS & 4 & $47.6(37.5)$ & $30.2(47.3)$ & $43.7(53.4)$ & $140.4(96.3)$ \\
S-tDCS & 4 & $19.9(22.8)$ & $32.5(12.7)$ & $21.4(55.2)$ & $15.8(41.6)$ \\
\hline
\end{tabular}

${ }^{1}$ Calculated only on two participants. Participant 8 refused to complete the probes; Participant 2 completed the probes, but they could not be scored because of failed recording equipment. Note: Percent gain calculated as the mean of three post-treatment probes or the mean of two 6-week follow-up probes minus the mean of three pre-treatment probes and subsequently divided by the mean of the three baseline probes. A-tDCS $=$ anodal, $\mathrm{C}-\mathrm{tDCS}=$ cathodal and S-tDCS $=$ sham .

\subsubsection{Secondary Outcome Measures}

WAB-R Language Quotient. The A-tDCS group demonstrated a clinically significant improvement (mean gain $\geq 5$ points) at both time intervals of post-treatment and follow-up; however, the C-tDCS group demonstrated clinically significant changes only at follow-up, not at post-treatment. The S-tDCS group did not meet criteria for clinically significant gains on the LQ for either time interval. At an individual level, all four participants from the A-tDCS group and two of four participants in both the C-tDCS and sham groups made clinically significant changes on the LQ at post-treatment and/or follow-up testing.

CETI. Caregivers of all four participants in the C-tDCS group, two of four participants in the S-tDCS group, and one of three participants in the A-tDCS group reported a clinically significant improvement in communication (mean gain $\geq 10$ points) at both post-treatment 
and follow-up assessments. All other participants in the A-tDCS and S-tDCS groups did not meet criteria for clinically significant gains on the CETI at either time interval. One participant (randomized to A-tDCS) did not have a significant other available to complete the CETI.

Oral Reading Accuracy and Rate. Mean percent gains in oral reading accuracy and rate are reported by group in Table 5. Overall, improvement in oral reading accuracy and rate occurred across all groups. Group means for the percent gain in oral reading accuracy between pre- and post-treatment were greater for the A-tDCS $(M=47.3 \%, \mathrm{SD}=34.7)$ and C-tDCS $(M=47.6 \%, \mathrm{SD}=37.5)$ groups than for the S-tDCS group $(M=19.9 \%, \mathrm{SD}=22.8)$; however, all three groups exhibited a more similar magnitude of gain at follow-up testing. Group means for the percent gain in oral reading rate $(\mathrm{wpm})$ were greater for the A-tDCS and C-tDCS groups than for the S-tDCS group at both post-treatment and follow-up. However, post-hoc non-parametric Kruskal-Wallis tests and Mann-Whitney U tests found no significant group differences on gains in accuracy or rate at post-treatment or follow-up.

Table 6. Individual scores and percent gain (effect size) in oral reading accuracy and rate (wpm) per the Naming and Oral Reading for Language in Aphasia 6-point scale (NORLA-6).

\begin{tabular}{|c|c|c|c|c|c|}
\hline Participant & $\begin{array}{c}\text { Polarity vs. } \\
\text { Sham }\end{array}$ & $\begin{array}{l}\text { Oral Reading } \\
\text { Accuracy Gain } \\
\text { (ES) at } \\
\text { Post-Treatment }\end{array}$ & $\begin{array}{c}\text { Oral Reading } \\
\text { Accuracy Gain } \\
\text { (ES) at Follow-Up } \\
\text { Testing }\end{array}$ & $\begin{array}{l}\text { Oral Reading } \\
\text { Rate Gain (ES) at } \\
\text { Post-Treatment }\end{array}$ & $\begin{array}{c}\text { Oral Reading } \\
\text { Rate Gain (ES) } \\
\text { at Follow-Up } \\
\text { Testing }\end{array}$ \\
\hline \multicolumn{6}{|l|}{ WAB-R AQ > 55} \\
\hline 1 & A-tDCS & $36.2(5.2)$ & $26.0(3.8)$ & $58.0(4.5)$ & $28.2(2.2)$ \\
\hline 2 & A-tDCS & $91.5(8.1)$ & NA & $47.7(9.5)$ & NA \\
\hline 3 & C-tDCS & $17.2(2.3)$ & $20.1(2.7)$ & $15.8(2.2)$ & 134.7 (18.9) \\
\hline 4 & C-tDCS & $89.5(4.8)$ & $92.0(5.0)$ & $13.7(0.7)$ & $110.3(5.0)$ \\
\hline 5 & S-tDCS & $40.0(6.0)$ & $27.8(4.2)$ & 67.7 (11.7) & $50.5(8.8)$ \\
\hline 6 & S-tDCS & $26.6(4.2)$ & $21.2(3.4)$ & $55.1(11.2)$ & $-27.3(-5.5)$ \\
\hline \multicolumn{6}{|l|}{ WAB-R AQ < 55} \\
\hline 7 & A-tDCS & $8.7(0.4)$ & $25.2(1.0)$ & $-5.0(0.0)$ & $455.0(14.2)$ \\
\hline 8 & A-tDCS & $52.5(5.8)$ & NA & $146.7(6.0)$ & NA \\
\hline 9 & C-tDCS & $14.5(0.7)$ & $30.8(1.6)$ & $124.0(6.7)$ & $353.6(19.0)$ \\
\hline 10 & C-tDCS & $69.3(2.4)$ & $-22.5(-0.8)$ & $20.8(0.4)$ & $31.3(0.6)$ \\
\hline 11 & S-tDCS & $25.7(2.4)$ & $30.1(2.8)$ & $16.5(2.2)$ & $1.2(0.2)$ \\
\hline 12 & S-tDCS & $-13.0(-5.4)$ & $50.4(21.2)$ & $-54.0(-1.6)$ & $-54.0(-1.6)$ \\
\hline
\end{tabular}

Note. Participant 8 refused to complete the follow-up probes; Participant 2 completed the follow-up probes but they could not be scored because of failed recording equipment. Percent gain calculated as the mean of three post-treatment probes or the mean of two 6-week follow-up probes minus the mean of three pre-treatment probes and subsequently divided by the mean of the three baseline probes. ES = effect size, calculated as the mean of three post-treatment probes or the mean of two 6-week follow-up probes minus the mean of three pre-treatment probes and subsequently divided by the standard deviation across the three baseline probes [42]. A clinically significant effect size was determined apriori to be greater than 3.9 (medium effect) $[44,45]$. NA $=$ not available. A-tDCS $=$ anodal, $\mathrm{C}$-tDCS $=$ cathodal, and S-tDCS $=$ sham .

Table 6 provides the percent change and effect sizes of NORLA- 6 averaged scores for oral reading accuracy and rate for each individual participant. Descriptive analyses of the percent of change on NORLA-6 accuracy and rate scores indicate wide variability across participants. Percent change was typically in the positive direction, except for Participant 12 who received S-tDCS and displayed a negative percent change for accuracy at posttreatment and for rate at both time intervals. Participant 6, who also received S-tDCS, exhibited a negative change for oral reading rate at follow-up testing, while Participant 10, who received C-tDCS, displayed a negative percent change for accuracy at followup. Individual effect sizes reaching clinical significance were distributed across all three treatment arms but were more representative of participants with less severe aphasia $(A Q>55)$ than for those with more severe aphasia $(A Q<55)$. 


\section{3. fMRI Cortical Activation Patterns}

Table 7 provides the neurophysiological data for each participant. With regard to perilesional activation, the three participants $(1,9$, and 11 ; each within a separate treatment arm) with the shortest time post-onset in the sample (range 6.2 to 6.3 months) did not demonstrate increased perilesional activation at post-treatment for any fMRI task. In contrast, the three participants $(8,10$, and 12; each within a separate treatment arm) who demonstrated increased perilesional activity across all tasks presented with time post-onset of 7.3, 9.2, and 18.6 months, respectively. The remaining six participants demonstrated increased perilesional activity only on some, but not all tasks ranged from 29.2 to 155.7 months post-onset.

Table 7. Number of activated voxels within a $5 \mathrm{~mm}$ area around the lesion (perilesional) and the ratio of activated voxels between the left vs. right hemisphere (left/right ratio) at pre- and post-treatment.

\begin{tabular}{|c|c|c|c|c|c|c|c|c|c|c|c|c|c|c|}
\hline \multirow{3}{*}{ Participant } & \multirow{3}{*}{$\begin{array}{c}\text { Polarity } \\
\text { vs. } \\
\text { Sham }\end{array}$} & \multirow{3}{*}{$\begin{array}{c}\text { Lesion } \\
\text { Size }(m L)\end{array}$} & \multicolumn{6}{|c|}{ Perilesional Activation } & \multicolumn{6}{|c|}{ Left/Right Ratio } \\
\hline & & & \multicolumn{2}{|c|}{ Task 1} & \multicolumn{2}{|c|}{ Task 2} & \multicolumn{2}{|c|}{ Task 3} & \multicolumn{2}{|c|}{ Task 1} & \multicolumn{2}{|c|}{ Task 2} & \multicolumn{2}{|c|}{ Task 3} \\
\hline & & & Pre & Post & Pre & Post & Pre & Post & Pre & Post & Pre & Post & Pre & Post \\
\hline \multicolumn{15}{|c|}{ WAB-R AQ > 55} \\
\hline 1 & A-tDCS & 66.9 & 57 & 0 & 0 & 0 & 135 & 122 & 0.74 & 0.56 & 0.91 & 0.76 & 0.56 & 0.86 \\
\hline 2 & A-tDCS & 80.3 & 339 & 92 & 86 & 6 & 142 & 284 & 0.75 & 0.71 & 0.68 & 4.31 & 0.47 & 0.70 \\
\hline 3 & C-tDCS & 92.8 & 816 & 375 & 45 & 2151 & 103 & 2244 & 0.68 & 0.75 & 0.51 & 1.53 & 0.81 & 1.60 \\
\hline 4 & C-tDCS & 94.7 & 34 & 81 & 317 & 114 & 357 & 434 & 0.76 & 0.86 & 0.35 & 0.66 & 0.58 & 0.65 \\
\hline 5 & S-tDCS & 76.6 & 768 & 641 & 181 & 117 & 216 & 600 & 0.45 & 0.69 & 0.35 & 0.44 & 0.53 & 0.71 \\
\hline 6 & S-tDCS & 155.8 & 238 & 238 & 24 & 0 & 154 & 412 & 0.67 & 0.43 & 0.12 & 0.17 & 0.39 & 0.81 \\
\hline \multicolumn{15}{|c|}{ WAB-R AQ $<55$} \\
\hline 7 & A-tDCS & 118.6 & 227 & 467 & NA & NA & NA & NA & 0.89 & 0.94 & NA & NA & NA & NA \\
\hline 8 & A-tDCS & 136.4 & 234 & 494 & 62 & 670 & 48 & 539 & 0.97 & 0.67 & 0.84 & 1.67 & 0.58 & 0.80 \\
\hline 9 & C-tDCS & 46.5 & 121 & 28 & 0 & 36 & 255 & 43 & 0.60 & 0.78 & 0.73 & 0.28 & 0.64 & 0.32 \\
\hline 10 & C-tDCS & 83.3 & 98 & 139 & 19 & 71 & 96 & 147 & 0.91 & 0.85 & 1.02 & 0.74 & 1.07 & 0.93 \\
\hline 11 & S-tDCS & 24.5 & 57 & 0 & 0 & 0 & 135 & 122 & 1.27 & 1.00 & 0.78 & 1.08 & 1.12 & 0.80 \\
\hline 12 & S-tDCS & 56.1 & 0 & 88 & 205 & 380 & 232 & 292 & 0.12 & 1.76 & 0.25 & 2.56 & 0.83 & 5.09 \\
\hline
\end{tabular}

Note. For Participant 7, tasks 2 and 3 resulted in a large number of movement artefacts at both pre- and post-treatment so these tasks were subsequently excluded from analysis. WAB-R AQ $=$ Western Aphasia Battery-Revised Aphasia Quotient [25]. Pre $=$ pre-treatment score, Post $=$ post-treatment score. A-tDCS $=$ anodal, C-tDCS $=$ cathodal, and S-tDCS $=$ sham. For Left $/$ Right Ratio: a value of 1 indicates equal activation in left vs. right hemisphere, a value $>1$ indicates more activity in the left hemisphere, and a value $<1$ indicates more activity in the right hemisphere. For perilesional activation, highlighted cells indicate increased perilesional activation at post-treatment. For left/right ratio, highlighted cells indicate increased left hemisphere activity from pre- to post-treatment.

Participants with less severe aphasia tended to exhibit increased activation of the left hemisphere from pre- to post-treatment relative to the right hemisphere, more so than those with more severe aphasia. The oral reading task (Task 2) and the imitation task (Task 3) were more likely to demonstrate increased activation in the left hemisphere from pre- to post-treatment relative to the right hemisphere, than the categorization task (Task 1).

\section{Discussion}

This study recorded stable vitals and no serious adverse events or unintended effects during, or up to six weeks after, an extended application of $1 \mathrm{~mA}$ tDCS applied to perilesional cortices for stroke survivors with nonfluent, chronic aphasia. These exploratory results are the first cohort study supporting the safety and tolerability of prolonged tDCS application (i.e., 30 sessions within a six-week interval) in aphasia rehabilitation. The literature has overall reported few adverse events with tDCS delivery, and those that have been reported are relatively mild (e.g., skin irritation, headache, dizziness, and nausea) $[10,51,52]$. It is notable that the risk of cerebral autoregulation or the reduction in cerebral blood flow following tDCS for patients with cerebrovascular diseases remains unknown [53]; however, recent meta-analyses found no significant difference in the occurrence of adverse events 
or in dropout rates between active tDCS and control arms in aphasia-focused investigations [10]. Nevertheless, safety and tolerability were important considerations for the present study as the completed protocol was beyond previous maximum durations of tDCS, i.e., 15 sessions within a three-week interval [54] or 14 sessions within an eight-week interval $[10,55]$. In addition to daily monitoring of vitals and physical side effects within all treatment sessions, we contacted participants weekly during the six-week follow-up interval to ensure that there were no subsequent side effects from the application of 30 doses of tDCS to a specific cortical region. These findings extend our knowledge about the safety limits of tDCS and add to its appeal as an adjuvant to SLT, especially as SLT is typically provided to persons with aphasia over a period of several months rather than isolated twoor three-week intervals.

As the current state of the science lacks consensus on the effectiveness of tDCS to improve communication in persons with aphasia beyond naming, the second aim of this study explored the effects of active tDCS and polarity on language performance and neurophysiology [10]. Specifically, we tested whether A-tDCS or C-tDCS (13 min at $1 \mathrm{~mA}$ ) delivered concurrently with an evidence-based SLT (90 min daily) for five days a week across six weeks results in greater communication gains and cortical activation than sham stimulation or the opposing polarity. Since participants received SLT within all treatment arms, we expected evident language improvement for all participants, especially in the context of high treatment intensities $[9,56]$. Beyond the expected gains attributed to behavioral SLT, participants receiving active tDCS (either anodal or cathodal) demonstrated greater gains on standardized language assessments that included all language modalities (auditory comprehension, oral expression, reading comprehension, and writing) than participants receiving sham stimulation. To our knowledge, this may be the first study to report the effects of $\mathrm{tDCS}$ on reading comprehension and written expression in aphasia. The same bolstering effect of active tDCS was also seen in caregivers reporting greater, and more frequently clinically significant, gains in daily communication on the CETI for participants receiving active versus sham stimulation. Similarly, participants receiving active tDCS demonstrated nonsignificant but larger gains on the trained oral reading probes than those receiving sham tDCS, although the groups were not matched on baseline performance and individual variability was evident. These results reinforce that $t D C S$, when paired with SLT, demonstrates potential for amplifying communication gains in persons with aphasia as evidenced across standardized, consumer-reported, and task-specific (i.e., oral reading) outcome measures [10].

The optimal dosage, intensity and frequency of tDCS, and its combination with speech and language therapy is still unclear with great variability across studies that have investigated the adjuvant effects of tDCS on aphasia treatment [10]. With few exceptions, studies have applied between 1 and $2 \mathrm{~mA}$ of anodal or cathodal tDCS for 10 to $20 \mathrm{~min}$ in 5 to 15 sessions delivered over a period of 1 to 3 weeks $[10,13]$. In our study we applied $1 \mathrm{~mA}$ tDCS for $13 \mathrm{~min}$. As indicated previously, these parameters were selected based on previous studies showing that the effects of $13 \mathrm{~min}$ of A-tDCS at $1 \mathrm{~mA}$ were maintained for up to $90 \mathrm{~min}$ post-stimulation [15]. Nevertheless, a weekly dose of $65 \mathrm{~min}$ of tDCS (i.e., $13 \mathrm{~min}, 5$ days/week) is less than the dose provided in many other studies. For example, in the largest double-blinded randomized clinical trial to date that has assessed the efficacy of adjunctive tDCS combined with aphasia therapy, Fridrikkson and colleagues applied $1 \mathrm{~mA}$ of anodal tDCS for $20 \mathrm{~min}$ over 15 sessions (i.e., $100 \mathrm{~min} /$ week for three weeks) [23]. However, when we compare the total dose of tDCS, our study provided $390 \mathrm{~min}$ of $\mathrm{tDCS}$ over the study period compared to a total of $300 \mathrm{~min}$ in the Fridrikkson study.

In addition to the type of behavioral treatment, it is similarly important to consider the dose of the behavioral treatment that is combined with the tDCS. In our study, each session provided 90 min of a combination of ORLA ${ }^{\circledR}$ and AphasiaScripts ${ }^{\circledR}$ for a total of 30 sessions over six weeks compared, for example, to the Fridrikkson study which provided $45 \mathrm{~min}$ of a naming treatment for 15 sessions over three weeks [23]. Given the high intensity of the behavioral treatment in our study, and research suggesting that high intensity treatment 
results in better functional communication outcomes than low intensity treatment [8], it is not possible to dissociate the effects of the tDCS itself from the effects of the behavioral treatment. Indeed, since all groups (anodal tDCS, cathodal tDCS, sham $\mathrm{tDCS}$ ) received the same intensive behavioral treatment, it is possible that differential effects of the tDCS polarities were masked by the effects of the behavioral treatment, especially on treatment probes that directly measure changes in performance on trained treatment stimuli.

Similar to the current literature, the data from the present study are not sufficient to declare superiority of one polarity versus another in aphasia rehabilitation. Clinically significant gains on the WAB-R AQ and CETI occurred with both polarities at post-treatment and follow-up endpoints; however, clinically significant gains on the CETI were more evident for individuals within the cathodal treatment arm. Clinically significant gains on the WAB-R LQ occurred for both cohorts at follow-up, yet only for the A-tDCS group at post-treatment. Gains in probe measures of oral reading accuracy and rate were noted for both active stimulation groups, with medium effect sizes achieved for some, but not all of the measures. Additionally, fMRI activation patterns demonstrated important polarity effects. C-tDCS to the left, lesioned hemisphere, which was expected to have an inhibitory effect, actually resulted in an increased number of activated voxels within both hemispheres and increased activation in perilesional regions. To date, investigations of cortical stimulation have largely supported a model of interhemispheric inhibition, even though some evidence suggests that unilateral injury can lead to cortical disinhibition, not only in the contralesional homotopic areas connected via the corpus callous, but also in neighboring ipsilesional cortices [57-59].

Notably, there was some inconsistency in the direction of change for the number of voxels activated across participants in the present study, with interesting trends of perilesional activation in relation to time post-stroke onset. Thus, supporting that bihemispheric or bimodal balance is dynamic even in chronic aphasia ( $>6$ months post-stroke) and is critical for functional recovery in aphasia, stimulation needs to be optimized for the individual patient [18]. Previous research suggests the activation of hemispheric and bihemispheric networks is a dynamic process that changes during the course of stroke recovery and is mediated by several factors including time from aphasia onset and specific task demands [60-62]. Typically, in the subacute phase, the right hemisphere exhibits stronger involvement in language functions with a subsequent shift towards increased left hemisphere activation in an apparent attempt to regain dominance within the chronic phase [63-65]. We postulate that the relative dominance of the right and left hemisphere roles was still evolving in the participants who were just beyond 6 months post-onset, and thus the window of opportunity to shift activation back to the left hemisphere had not yet opened and precluded the up-regulation of perilesional activation regardless of the treatment provided.

We also suggest that C-tDCS may not always serve an inhibitory role, and in some instances, may be excitatory like A-tDCS. For example, Batsikadze and colleagues compared the effects of $1 \mathrm{~mA}$ and $2 \mathrm{~mA}$ tDCS on cortical excitability when using $35 \mathrm{~cm}^{2}$ electrodes [66]. They found that $1 \mathrm{~mA}$ cathodal tDCS decreased corticospinal excitability, whereas $2 \mathrm{~mA}$ cathodal tDCS resulted in a significant increase of motor evoked potential amplitudes. While an enhancement of tDCS intensity does not necessarily increase efficacy of stimulation, it may shift the direction of excitability and this should be considered when using different intensities and durations of tDCS [66]. Although we administered $1 \mathrm{~mA}$ of C-tDCS in our study, we used a smaller sized electrode $\left(16 \mathrm{~cm}^{2}\right)$. Smaller electrodes may produce more focal current density and could lead to more effective and localized neural modulation than larger ones $[67,68]$. In our study, the smaller electrodes resulted in a current density of $0.063 \mathrm{~mA} / \mathrm{cm}^{2}$ that more closely resembled, and even exceeded, the current density of a $35 \mathrm{~cm}^{2}$ electrode and $2 \mathrm{~mA}$ of C-tDCS (i.e., $0.057 \mathrm{~mA} / \mathrm{cm}^{2}$ ), thereby possibly shifting the polarity from inhibitory to excitatory.

Long-term potentiation and the maintenance of behavioral treatment gains are critical for aphasia rehabilitation. Across all three standardized measures (WAB-R AQ, WAB-R 
LQ, and CETI), both conditions receiving active tDCS (anodal and cathodal) continued to exhibit clinically significant gains compared to baseline performance following a six-week hiatus from treatment. Lasting improvements in language may suggest that tDCS in conjunction with brain-derived neurotrophic factor (BDNF) plays a role in enhanced neural plasticity, with more robust long-term learning and efficient reorganization of neural circuits $[69,70]$. Future research is required to investigate the neurophysiological mechanisms contributing to long-term potentiation during aphasia recovery, and specifically which of those mechanisms are responsive to tDCS, increase synaptic efficacy, and reestablish healthy interhemispheric interactions [69,71-73].

We have reported here 12 participants who were randomized to receive anodal, cathodal, or sham stimulation who differed on many variables including lesion size and location. The heterogeneity of lesion and aphasia characteristics are well-known in stroke rehabilitation fields; however, they serve as limitations for study recruitment and generalization of the present findings. To address heterogeneity, a neuronavigation system was used to individualize electrode placement and optimize the effects of the tDCS; however, it resulted in quite disparate electrode placement sites. It is not clear whether or not the fMRIguided localization of stimulation site served to normalize the effects of the tDCS across individuals. Furthermore, recent computational models have illustrated the complexity of factors contributing to the distribution of current flow through the brain, which may have added further, and at this time indistinguishable, variance into our outcome measures $[12,21,22,74-76]$. Future research may seek to further isolate or account for potentially mediating variables such as time post-aphasia onset by defining more narrow windows of recovery and by more closely matching subjects with regard to lesion location and size.

\section{Conclusions}

The results support that tDCS (delivered to the perilesional cortex at $1 \mathrm{~mA}$ for $13 \mathrm{~min}$, five days a week for six weeks) is safe and well tolerated. Further investigation of prolonged, programmatic applications of tDCS combined with behavioral SLT to support aphasia recovery in stroke survivors is warranted based on demonstrated safety, improved language compared to sham stimulation, and increased cortical activation.

Author Contributions: Conceptualization, L.R.C.; data curation, E.M.B., X.W. and L.L.P.; formal analysis, L.R.C., X.W. and L.L.P.; funding acquisition, L.R.C.; investigation, L.R.C., E.M.B., X.W. and L.L.P.; methodology, L.R.C., E.M.B. and X.W.; project administration, L.R.C.; resources, L.R.C.; software, L.R.C.; supervision, L.R.C.; validation, L.R.C., E.M.B., X.W. and L.L.P.; visualization, L.R.C., E.M.B. and L.L.P.; writing-original draft, L.R.C. and L.L.P.; writing-review and editing, L.R.C., E.M.B., X.W. and L.L.P. All authors have read and agreed to the published version of the manuscript.

Funding: This research was funded by the National Institute on Deafness and Other Communication Disorders award number 5R21DC009876-02 (to L.R.C.). The content is solely the responsibility of the authors and does not necessarily represent the official views of the National Institute on Deafness and Other Communication Disorders or the National Institutes of Health. Additional support has been provided by the Thoma Foundation.

Institutional Review Board Statement: The study was conducted according to the guidelines of the Declaration of Helsinki and approved by the Institutional Review Board at Northwestern University (STU00011132). It is registered as a clinical trial (NCT01486654).

Informed Consent Statement: Informed consent was obtained from all subjects involved in the study.

Acknowledgments: We would like to acknowledge the following study team members whose contributions and scholarship led to the success of this study: Rosalind Hurwitz, Todd Parrish, Lynn Rogers, James Stinear, Richard Harvey, Kenta Wakaizumi, Marwan Baliki, Julia Carpenter, and Roz Kaye.

Conflicts of Interest: The authors declare no conflict of interest. The funders had no role in the design of the study; in the collection, analyses, or interpretation of data; in the writing of the manuscript, or in the decision to publish the results. 


\section{References}

1. Avan, A.; Digaleh, H.; Di Napoli, M.; Stranges, S.; Behrouz, R.; Shojaeianbabaei, G.; Amiri, A.; Tabrizi, R.; Mokhber, N.; Spence, J.D.; et al. Socioeconomic status and stroke incidence, prevalence, mortality, and worldwide burden: An ecological analysis from the Global Burden of Disease Study 2017. BMC Med. 2019, 17, 1-30. [CrossRef]

2. Flowers, H.L.; Silver, F.L.; Fang, J.; Rochon, E.; Martino, R. The incidence, co-occurrence, and predictors of dysphagia, dysarthria, and aphasia after first-ever acute ischemic stroke. J. Commun. Disord. 2013, 46, 238-248. [CrossRef]

3. Flowers, H.L.; Skoretz, S.A.; Silver, F.L.; Rochon, E.; Fang, J.; Flamand-Roze, C.; Martino, R. Poststroke aphasia frequency, recovery, and outcomes: A systematic review and meta-analysis. Arch. Phys. Med. Rehabil. 2016, 97, 2188-2201.e8. [CrossRef]

4. $\quad$ Dalemans, R.J.P.; De Witte, L.P.; Wade, D.; Heuvel, W.V.D. Social participation through the eyes of people with aphasia. Int. J. Lang. Commun. Disord. 2010, 45, 537-550. [CrossRef]

5. Hilari, K.; Northcott, S.; Roy, P.; Marshall, J.; Wiggins, R.D.; Chataway, J.; Ames, D. Psychological distress after stroke and aphasia: The first six months. Clin. Rehabil. 2010, 24, 181-190. [CrossRef]

6. Bullier, B.; Cassoudesalle, H.; Villain, M.; Cogné, M.; Mollo, C.; De Gabory, I.; Dehail, P.; Joseph, P.-A.; Sibon, I.; Glize, B. New factors that affect quality of life in patients with aphasia. Ann. Phys. Rehabil. Med. 2020, 63, 33-37. [CrossRef]

7. Ashaie, S.A.; Hurwitz, R.; Cherney, L.R. Depression and subthreshold depression in stroke-related aphasia. Arch. Phys. Med. Rehabil. 2019, 100, 1294-1299. [CrossRef]

8. Brady, M.C.; Kelly, H.; Godwin, J.; Enderby, P.; Campbell, P. Speech and language therapy for aphasia following stroke. Cochrane Database Syst. Rev. 2016, CD000425. [CrossRef]

9. Cherney, L.R.; Patterson, J.P.; Raymer, A.; Frymark, T.; Schooling, T. Evidence-based systematic review: Effects of intensity of treatment and constraint-induced language therapy for individuals with stroke-induced aphasia. J. Speech Lang. Hear. Res. 2008, 51, 1282-1299. [CrossRef]

10. Elsner, B.; Kugler, J.; Pohl, M.; Mehrholz, J. Transcranial direct current stimulation (tDCS) for improving aphasia in adults with aphasia after stroke. Cochrane Database Syst. Rev. 2019, 2019, CD009760. [CrossRef]

11. Lefaucheur, J.-P.; Antal, A.; Ayache, S.S.; Benninger, D.H.; Brunelin, J.; Cogiamanian, F.; Cotelli, M.; De Ridder, D.; Ferrucci, R.; Langguth, B.; et al. Evidence-based guidelines on the therapeutic use of transcranial direct current stimulation ( $t D C S$ ). Clin. Neurophysiol. 2017, 128, 56-92. [CrossRef] [PubMed]

12. Ulanov, M.; Shtyrov, Y.; Stroganova, T. Transcranial direct current stimulation as a tool to induce language recovery in patients with post-stroke aphasia. Neurosci. Behav. Physiol. 2019, 49, 1169-1180. [CrossRef]

13. Marangolo, P. The potential effects of transcranial direct current stimulation (tDCS) on language functioning: Combining neuromodulation and behavioral intervention in aphasia. Neurosci. Lett. 2020, 719, 133329. [CrossRef] [PubMed]

14. Nitsche, M.A.; Paulus, W. Excitability changes induced in the human motor cortex by weak transcranial direct current stimulation. J. Physiol. 2000, 527, 633-639. [CrossRef] [PubMed]

15. Nitsche, M.A.; Paulus, W. Sustained excitability elevations induced by transcranial DC motor cortex stimulation in humans. Neurology 2001, 57, 1899-1901. [CrossRef]

16. Nitsche, M.A.; Nitsche, M.S.; Klein, C.C.; Tergau, F.; Rothwell, J.C.; Paulus, W. Level of action of cathodal DC polarisation induced inhibition of the human motor cortex. Clin. Neurophysiol. 2003, 114, 600-604. [CrossRef]

17. Kiran, S. What is the nature of poststroke language recovery and reorganization? ISRN Neurol. 2012, 2012, 786872. [CrossRef]

18. Di Pino, G.; Pellegrino, G.; Assenza, G.; Capone, F.; Ferreri, F.; Formica, D.; Ranieri, F.; Tombini, M.; Ziemann, U.; Rothwell, J.C.; et al. Modulation of brain plasticity in stroke: A novel model for neurorehabilitation. Nat. Rev. Neurol. 2014, 10, 597-608. [CrossRef] [PubMed]

19. Monti, A.; Cogiamanian, F.; Marceglia, S.; Ferrucci, R.; Mameli, F.; Mrakic-Sposta, S.; Vergari, M.; Zago, S.; Priori, A. Improved naming after transcranial direct current stimulation in aphasia. J. Neurol. Neurosurg. Psychiatry 2008, 79, 451-453. [CrossRef]

20. Shah-Basak, P.P.; Norise, C.; Garcia, G.; Torres, J.; Faseyitan, O.; Hamilton, R.H. Individualized treatment with transcranial direct current stimulation in patients with chronic non-fluent aphasia due to stroke. Front. Hum. Neurosci. 2015, 9, 201. [CrossRef]

21. Datta, A.; Baker, J.M.; Bikson, M.; Fridriksson, J. Individualized model predicts brain current flow during transcranial directcurrent stimulation treatment in responsive stroke patient. Brain Stimul. 2011, 4, 169-174. [CrossRef]

22. Galletta, E.E.; Cancelli, A.; Cottone, C.; Simonelli, I.; Tecchio, F.; Bikson, M.; Marangolo, P. Use of computational modeling to inform tDCS electrode montages for the promotion of language recovery in post-stroke aphasia. Brain Stimul. 2015, 8, 1108-1115. [CrossRef] [PubMed]

23. Fridriksson, J.; Rorden, C.; Elm, J.; Sen, S.; George, M.S.; Bonilha, L. Transcranial direct current stimulation vs sham stimulation to treat aphasia after stroke: A randomized clinical trial. JAMA Neurol. 2018, 75, 1470-1476. [CrossRef] [PubMed]

24. Alharbi, M.F.; Armijo-Olivo, S.; Kim, E.S. Transcranial direct current stimulation (tDCS) to improve naming ability in post-stroke aphasia: A critical review. Behav. Brain Res. 2017, 332, 7-15. [CrossRef] [PubMed]

25. Kertesz, A. Western Aphasia Battery (Revised); PsychCorp: San Antonio, TX, USA, 2007.

26. Oldfield, R. The assessment and analysis of handedness: The Edinburgh inventory. Neuropsychology 1971, 9, 97-113. [CrossRef]

27. Cherney, L.R.; Erickson, R.K.; Small, S.L. Epidural cortical stimulation as adjunctive treatment for non-fluent aphasia: Preliminary findings. J. Neurol. Neurosurg. Psychiatry 2010, 81, 1014-1021. [CrossRef]

28. Cherney, L.R.; Small, S.L. Task-dependent changes in brain activation following therapy for nonfluent aphasia: Discussion of two individual cases. J. Int. Neuropsychol. Soc. 2006, 12, 828-842. [CrossRef] 
29. Cherney, L.R.; Babbitt, E.M.; Hurwitz, R.; Rogers, L.M.; Stinear, J.; Wang, X.; Harvey, R.L.; Parrish, T. Transcranial direct current stimulation and aphasia: The case of Mr. C. Top. Stroke Rehabil. 2013, 20, 5-20. [CrossRef]

30. Nitsche, M.A.; Cohen, L.G.; Wassermann, E.M.; Priori, A.; Lang, N.; Antal, A.; Paulus, W.; Hummel, F.; Boggio, P.S.; Fregni, F.; et al. Transcranial direct current stimulation: State of the art 2018. Brain Stimul. 2008, 1, 206-223. [CrossRef] [PubMed]

31. Nitsche, M.A.; Liebetanz, D.; Lang, N.; Antal, A.; Tergau, F.; Paulus, W. Safety criteria for transcranial direct current stimulation (tDCS) in humans. Clin. Neurophysiol. 2003, 114, 2220-2222. [CrossRef]

32. Bikson, M.; Grossman, P.; Thomas, C.; Zannou, A.L.; Jiang, J.; Adnan, T.; Mourdoukoutas, A.P.; Kronberg, G.; Truong, D.; Boggio, P.; et al. Safety of transcranial direct current stimulation: Evidence based update 2016. Brain Stimul. 2016, 9, 641-661. [CrossRef] [PubMed]

33. Cherney, L.R.; Merbitz, C.T.; Grip, J.C. Efficacy of oral reading in aphasia treatment outcome. Rehabil. Lit. 1986, 47, 112-118. [PubMed]

34. Cherney, L.R. Efficacy of oral reading in the treatment of two patients with chronic Broca's aphasia. Top. Stroke Rehabil. 1995, 2, 57-67. [CrossRef]

35. Cherney, L.R. Oral reading for language in aphasia (ORLA): Evaluating the efficacy of computer-delivered therapy in chronic nonfluent aphasia. Top. Stroke Rehabil. 2010, 17, 423-431. [CrossRef]

36. Cherney, L.R.; Halper, A.S.; Holland, A.L.; Cole, R. Computerized script training for aphasia: Preliminary results. Am. J. Speech-Language Pathol. 2008, 17, 19-34. [CrossRef]

37. Lee, J.B.; Kaye, R.C.; Cherney, L.R. Conversational script performance in adults with non-fluent aphasia: Treatment intensity and aphasia severity. Aphasiology 2009, 23, 885-897. [CrossRef]

38. Manheim, L.M.; Halper, A.S.; Cherney, L. Patient-reported changes in communication after computer-based script training for aphasia. Arch. Phys. Med. Rehabil. 2009, 90, 623-627. [CrossRef] [PubMed]

39. Wallace, S.J.; Worrall, L.; Rose, T.; Le Dorze, G.; Breitenstein, C.; Hilari, K.; Babbitt, E.; Bose, A.; Brady, M.; Cherney, L.R.; et al. A core outcome set for aphasia treatment research: The ROMA consensus statement. Int. J. Stroke 2019, 14, 180-185. [CrossRef]

40. Lomas, J.; Pickard, L.; Bester, S.; Elbard, H.; Finlayson, A.; Zoghaib, C. The communicative effectiveness index: Development and psychometric evaluation of a functional communication measure for adult aphasia. J. Speech Hear. Disord. 1989, 54, 113-124. [CrossRef]

41. Gilmore, N.; Dwyer, M.; Kiran, S. Benchmarks of significant change after aphasia rehabilitation. Arch. Phys. Med. Rehabil. 2019, 100, 1131-1139.e87. [CrossRef]

42. Pitts, L.L.; Hurwitz, R.; Lee, J.B.; Carpenter, J.; Cherney, L.R. Validity, reliability and sensitivity of the NORLA-6: Naming and oral reading for language in aphasia 6-point scale. Int. J. Speech-Language Pathol. 2017, 20, 274-283. [CrossRef]

43. Katz, R.C.; Wertz, R.T. The efficacy of computer-provided reading treatment for chronic aphasic adults. J. Speech Lang. Hear. Res. 1997, 40, 493-507. [CrossRef] [PubMed]

44. Beeson, P.M.; Robey, R.R. Evaluating single-subject treatment research: Lessons learned from the aphasia literature. Neuropsychol. Rev. 2006, 16, 161-169. [CrossRef]

45. Robey, R.R.; Schultz, M.C.; Crawford, A.B.; Sinner, C.A. Single-subject clinical-outcome research: Designs, data, effect sizes, and analyses. Aphasiology 1999, 13, 445-473. [CrossRef]

46. Robey, R.R. A meta-analysis of clinical outcomes in the treatment of aphasia. J. Speech Lang. Hear. Res. 1998, 41, 172-187. [CrossRef] [PubMed]

47. Fischl, B.; Sereno, M.I.; Dale, A.M. Cortical surface-based analysis: II: Inflation, flattening, and a surface-based coordinate system. Neuroimage 1999, 9, 195-207. [CrossRef]

48. Greve, D.N.; Fischl, B. Accurate and robust brain image alignment using boundary-based registration. NeuroImage 2009, 48, 63-72. [CrossRef]

49. Jenkinson, M.; Smith, S. A global optimisation method for robust affine registration of brain images. Med. Image Anal. 2001, 5, 143-156. [CrossRef]

50. Jenkinson, M.; Bannister, P.; Brady, M.; Smith, S. Improved optimization for the robust and accurate linear registration and motion correction of brain images. NeuroImage 2002, 17, 825-841. [CrossRef]

51. Brunoni, A.R.; Amadera, J.; Berbel, B.; Volz, M.S.; Rizzerio, B.G.; Fregni, F. A systematic review on reporting and assessment of adverse effects associated with transcranial direct current stimulation. Int. J. Neuropsychopharmacol. 2011, 14, 1133-1145. [CrossRef]

52. Poreisz, C.; Boros, K.; Antal, A.; Paulus, W. Safety aspects of transcranial direct current stimulation concerning healthy subjects and patients. Brain Res. Bull. 2007, 72, 208-214. [CrossRef]

53. List, J.; Lesemann, A.; Kübke, J.C.; Külzow, N.; Schreiber, S.J.; Flöel, A. Impact of tDCS on cerebral autoregulation in aging and in patients with cerebrovascular diseases. Neurology 2015, 84, 626-628. [CrossRef]

54. Polanowska, K.; Leśniak, M.; Seniów, J. P 114.Anodal transcranial direct current stimulation in early treatment of post-stroke non-fluent aphasia. Clin. Neurophysiol. 2013, 124, e118-e119. [CrossRef]

55. Duncan, E.S.; Nakkawita, S.G. Clinical feasibility of combining transcranial direct current stimulation with standard aphasia therapy. Ann. Indian Acad. Neurol. 2020, 23, S102-S108.

56. Cherney, L.R.; Patterson, J.P.; Raymer, A.M. Intensity of aphasia therapy: Evidence and efficacy. Curr. Neurol. Neurosci. Rep. 2011, 11, 560-569. [CrossRef] 
57. Bütefisch, C.M.; Kleiser, R.; Seitz, R.J. Post-lesional cerebral reorganisation: Evidence from functional neuroimaging and transcranial magnetic stimulation. J. Physiol. 2006, 99, 437-454. [CrossRef] [PubMed]

58. Lang, N.; Nitsche, M.A.; Paulus, W.; Rothwell, J.C.; Lemon, R.N. Effects of transcranial direct current stimulation over the human motor cortex on corticospinal and transcallosal excitability. Exp. Brain Res. 2004, 156, 439-443. [CrossRef] [PubMed]

59. Shimizu, T.; Hosaki, A.; Hino, T.; Sato, M.; Komori, T.; Hirai, S.; Rossini, P.M. Motor cortical disinhibition in the unaffected hemisphere after unilateral cortical stroke. Brain 2002, 125, 1896-1907. [CrossRef]

60. Finger, S.; Buckner, R.L.; Buckingham, H. Does the right hemisphere take over after damage to Broca's area? the Barlow case of 1877 and its history. Brain Lang. 2003, 85, 385-395. [CrossRef]

61. Hillis, A.E.; Seeley, W.W.; Marty, F.M.; Holmes, T.M.; Upchurch, K.; Soiffer, R.J.; Antin, J.H.; Baden, L.R.; Bromfield, E.B. Aphasia: Progress in the last quarter of a century. Neurology 2007, 69, 200-213. [CrossRef]

62. Kiran, S.; Meier, E.L.; Johnson, J.P. Neuroplasticity in aphasia: A proposed framework of language recovery. J. Speech Lang. Hear. Res. 2019, 62, 3973-3985. [CrossRef] [PubMed]

63. Saur, D.; Lange, R.; Baumgaertner, A.; Schraknepper, V.; Willmes, K.; Rijntjes, M.; Weiller, C. Dynamics of language reorganization after stroke. Brain 2006, 129, 1371-1384. [CrossRef] [PubMed]

64. Winhuisen, L.; Thiel, A.; Schumacher, B.; Kessler, J.; Rudolf, J.; Haupt, W.F.; Heiss, W.D. Role of the contralateral inferior frontal gyrus in recovery of language function in poststroke aphasia: A combined repetitive transcranial magnetic stimulation and positron emission tomography study. Stroke 2005, 36, 1759-1763. [CrossRef]

65. Winhuisen, L.; Thiel, A.; Schumacher, B.; Kessler, J.; Rudolf, J.; Haupt, W.F.; Heiss, W.D. The right inferior frontal gyrus and poststroke aphasia: A follow-up investigation. Stroke 2007, 38, 1286-1292. [CrossRef] [PubMed]

66. Batsikadze, G.; Moliadze, V.; Paulus, W.; Kuo, M.-F.; Nitsche, M.A. Partially non-linear stimulation intensity-dependent effects of direct current stimulation on motor cortex excitability in humans. J. Physiol. 2013, 591, 1987-2000. [CrossRef]

67. Solomons, C.D.; Shanmugasundaram, V. Transcranial direct current stimulation: A review of electrode characteristics and materials. Med. Eng. Phys. 2020, 85, 63-74. [CrossRef]

68. Bastani, A.; Jaberzadeh, S. a-tDCS differential modulation of corticospinal excitability: The effects of electrode size. Brain Stimul. 2013, 6, 932-937. [CrossRef] [PubMed]

69. Fritsch, B.; Reis, J.; Martinowich, K.; Schambra, H.M.; Ji, Y.; Cohen, L.G.; Lu, B. Direct current stimulation promotes BDNFdependent synaptic plasticity: Potential implications for motor learning. Neuron 2010, 66, 198-204. [CrossRef] [PubMed]

70. Fridriksson, J.; Elm, J.; Stark, B.C.; Basilakos, A.; Rorden, C.; Sen, S.; George, M.S.; Gottfried, M.; Bonilha, L. BDNF genotype and tDCS interaction in aphasia treatment. Brain Stimul. 2018, 11, 1276-1281. [CrossRef] [PubMed]

71. Brunoni, A.R.; Nitsche, M.A.; Bolognini, N.; Bikson, M.; Wagner, T.; Merabet, L.; Edwards, D.J.; Valero-Cabre, A.; Rotenberg, A.; Pascual-Leone, A.; et al. Clinical research with transcranial direct current stimulation (tDCS): Challenges and future directions. Brain Stimul. 2012, 5, 175-195. [CrossRef]

72. Warburton, E.; Price, C.J.; Swinburn, K.; Wise, R.J.S. Mechanisms of recovery from aphasia: Evidence from positron emission tomography studies. J. Neurol. Neurosurg. Psychiatry 1999, 66, 155-161. [CrossRef] [PubMed]

73. Biou, E.; Cassoudesalle, H.; Cogné, M.; Sibon, I.; De Gabory, I.; Dehail, P.; Aupy, J.; Glize, B. Transcranial direct current stimulation in post-stroke aphasia rehabilitation: A systematic review. Ann. Phys. Rehabil. Med. 2019, 62, 104-121. [CrossRef] [PubMed]

74. Schmidt, C.; Wagner, S.; Burger, M.; Van Rienen, U.; Wolters, C. Impact of uncertain head tissue conductivity in the optimization of transcranial direct current stimulation for an auditory target. J. Neural Eng. 2015, 12, 046028. [CrossRef] [PubMed]

75. Datta, A.; Bikson, M.; Fregni, F. Transcranial direct current stimulation in patients with skull defects and skull plates: Highresolution computational FEM study of factors altering cortical current flow. NeuroImage 2010, 52, 1268-1278. [CrossRef] [PubMed]

76. Wagner, T.; Fregni, F.; Eden, U.; Ramos-Estebanez, C.; Grodzinsky, A.; Zahn, M.; Pascual-Leone, A. Transcranial magnetic stimulation and stroke: A computer-based human model study. NeuroImage 2006, 30, 857-870. [CrossRef] [PubMed] 